\title{
Exotic lepton-flavor violating Higgs decays
}

\author{
Jared A. Evans, ${ }^{a}$ Philip Tanedo ${ }^{b}$ and Mohammadreza Zakeri $^{c}$ \\ ${ }^{a}$ Department of Physics, University of Cincinnati, \\ OH 45221, U.S.A. \\ ${ }^{b}$ Department of Physics $\&$ Astronomy, University of California, \\ Riverside, CA 92521, U.S.A. \\ ${ }^{c}$ Institute of Theoretical Physics, Chinese Academy of Sciences, \\ Beijing 100190, China \\ E-mail: jaredaevans@gmail.com, flip.tanedo@ucr.edu, mzake001@ucr.edu
}

ABSTRACT: Exotic Higgs decays are promising channels to discover new physics in the near future. We present a simple model with a new light scalar that couples to the Standard Model through a charged lepton-flavor violating interaction. This can yield exciting new signatures, such as $h \rightarrow e^{+} e^{+} \mu^{-} \mu^{-}$, that currently have no dedicated searches at the Large Hadron Collider. We discuss this model in detail, assess sensitivity from flavor constraints, explore current constraints from existing multi-lepton searches, and construct a new search strategy to optimally target these exotic, lepton-flavor violating Higgs decays.

KEYWords: Phenomenological Models

ARXIV EPRINT: 1910.07533 


\section{Contents}

1 Introduction 1

2 A simplified model of lepton-flavor violating mediators 3

2.1 Low-energy couplings to leptons 3

2.2 Gauge-invariant effective theory 4

3 Flavor constraints 4

3.1 Pure off-diagonal, single coupling 5

$\begin{array}{ll}3.2 \text { Multi-coupling } & 6\end{array}$

4 Collider $\quad 7$

4.1 Multi-lepton search limits $\quad 9$

$\begin{array}{ll}4.2 \text { A dedicated Higgs search } & 12\end{array}$

$\begin{array}{lll}4.3 & \text { Long-lived LFV scalars } & 15\end{array}$

$\begin{array}{lll}5 & \text { Conclusion } & 17\end{array}$

$\begin{array}{ll}\text { A Renormalizable ultraviolet completions } & 18\end{array}$

$\begin{array}{ll}\text { A.1 Vector-like leptons } & 18\end{array}$

$\begin{array}{ll}\text { A.2 Froggatt-Nielsen } & 18\end{array}$

$\begin{array}{lll}\text { A.3 } & \text { R-parity violating supersymmetry } & 19\end{array}$

$\begin{array}{ll}\text { B Review of chiral structure } & 19\end{array}$

\section{Introduction}

The Large Hadron Collider (LHC) has produced millions of Higgs bosons since the 2012 discovery by the ATLAS and CMS collaborations [1,2]. This growing sample allows for searches probing exotic Higgs decays, which may provide the pathway to uncovering new physics [3]. Since the Higgs vacuum expectation value (VEv) is the order parameter of electroweak symmetry breaking, the Higgs field has a special role in the possible ways that the Standard Model can couple to otherwise inaccessible new particles. Our current data about the Higgs sector may easily accommodate an $\mathcal{O}(10 \%)$ branching fraction into exotic final states. In fact, many possible exotic final states could be discoverable even if only a few Higgses decay that way over the lifetime of the LHC, e.g. [3, 4].

Hidden sectors [5] can provide a very simple origin for an exotic Higgs decay. One of the most compelling reasons to consider the addition of a hidden sector onto the Standard Model is dark matter. For example, dark matter may interact with visible matter through a lower-mass mediator [6-8]. The dark matter-mediator coupling can be fixed 
to yield the observed dark matter abundance through thermal freeze out. On the other hand, the couplings of the mediator to visible matter only needs to be large enough to account for the observed dark matter relic abundance. This can easily be small enough to avoid laboratory-based experimental bounds $[9,10]$. Independent of any connection to dark matter, the possible existence of low-mass particles that interact weakly with visible matter may be tested through their interactions with the Higgs. In fact, for many lowenergy coupling structures such a connection to the Higgs may be mandated by electroweak gauge invariance.

The discovery of lepton flavor violating (LFV) couplings in the charged lepton sector would be a striking indication of physics beyond the Standard Model. The possibility of additional LFV contributions beyond neutrino mixing is especially tantalizing in the wake of various flavor physics anomalies involving leptons. Among these anomalies are the lepton flavor universality violation in $R_{K^{(*)}}$ and $R_{D^{(*)}}$ [11, 12], the proton radius puzzle [13], and the long standing anomalous magnetic moment of the muon [14]. All of these have received various hidden sector explanations, see e.g. [15-20]. There are, of course, many other observables that have so far proven consistent with Standard Model expectations, such as muonium oscillation, $\mu^{-} \rightarrow e^{-} e^{+} e^{-}$[21], or $\tau \rightarrow \mu \gamma$ [22], that provide stringent constraints on possible new physics.

A largely unexplored scenario is that of a light, spin-0 particle $\varphi$ that interacts with the Standard Model through LFV couplings to charged leptons [23]. This has been studied [23] in the context of fitting the $\gamma$-ray excess in the galactic center [24-41]. ${ }^{1}$ When this scalar is lighter than the Higgs, it is possible for the Higgs to decay through an exotic four-lepton channel, where $h \rightarrow \ell^{+} \ell^{\prime-} \varphi \rightarrow 4 \ell$ or $h \rightarrow \varphi^{*} \varphi \rightarrow 4 \ell$. In the case of prompt $\varphi$ decays, this exciting signature does not yet have any dedicated search. Existing searches for displaced vertices probe down to $\mathcal{O}(100 \mu \mathrm{m})$, corresponding to coupling strengths $\lesssim 10^{-6}$ for an $10 \mathrm{GeV}$ mediator; however, they are not optimized for this signature. Further, despite the simplicity of the model, there are exotic four-lepton signatures, e.g. $e^{+} e^{+} \mu^{-} \mu^{-}$, that can naturally emerge easily, but that have thus far skirted systematic signature classification programs, see e.g. [3, 44, 45].

In addition to the potential collider signatures, this model of a scalar with LFV couplings could explain the outstanding discrepancy in the muon anomalous magnetic moment $[14,23]$. A separate dedicated collider study of this model focused on a mediator that is heavier than dark matter and the decay of the Standard Model-like Higgs boson to $\tau \mu$ [46]. Recent complementary studies have explored displaced vertices [47] and signals at future $e^{+} e^{-}$colliders [48]. We note that our focus is distinct from the extensively studied flavorful dark sector scenarios in which both the mediator and dark matter carry flavor charges, e.g. [49-52].

In this paper we examine the collider phenomenology of a scalar that is produced in decays of the Higgs and then subsequently decays through an exotic LFV coupling. The low-energy model is detailed in section 2. Section 3 explores flavor constraints both when

\footnotetext{
${ }^{1}$ For the current status of this anomaly, we refer to recent statistical analyses supporting either a possible dark matter [42] or point source [43] interpretation. The proposed collider search in this manuscript is independent of the ultimate interpretation of the $\gamma$-ray excess.
} 
only a single off-diagonal coupling is present, and when multiple couplings are present simultaneously. Collider signatures are explored in section 4. To ascertain the current constraints, we present a phenomenological study of this signal based on a recast of a CMS search for exotic multi-lepton final states [53] in section 4.1. We then design a dedicated search that would capitalize on the unique kinematics to greatly enhance sensitivity to the model in section 4.2. We comment on long-lived decays in section 4.3 and conclude in section 5. We also provide several simple UV completions for the low-energy theory in appendix A, and review the chiral structure of these couplings in appendix B.

\section{A simplified model of lepton-flavor violating mediators}

We focus on the flavor-violating couplings of charged leptons to a gauge singlet scalar mediator, $\varphi$. This mediator may be real or complex. In this section, we discuss the lowenergy model and the electroweak gauge-invariant effective theory. Appendix A describes some simple UV completions.

\section{$2.1 \quad$ Low-energy couplings to leptons}

For this study, we make the reasonable assumption that any additional hidden sector particles, e.g. dark matter, do not influence the multi-lepton Higgs decays of interest. This can be accomplished easily if, for instance, the other dark sector particles are heavier than the mediator. The interactions of $\varphi$ with charged leptons $\ell$ are encoded in the effective Lagrangian terms,

$$
\mathcal{L} \supset\left(y_{i j} \bar{\ell}_{i} P_{L} \ell_{j} \varphi+y_{i j}^{*} \bar{\ell}_{j} P_{R} \ell_{i} \varphi^{*}\right)+\left(y_{i j}^{\prime} \bar{\ell}_{i} P_{R} \ell_{j} \varphi+y_{i j}^{\prime *} \bar{\ell}_{j} P_{L} \ell_{i} \varphi^{*}\right)
$$

where $i, j$ index charged lepton flavor mass eigenstates $(1=e, 2=\mu, 3=\tau)$. See appendix B for a brief review of the relevant chiral structure. The terms within each parenthesis are related by complex conjugation. When $\varphi$ is real, the $y^{\prime}$ terms are redundant and should be removed. Throughout most of this work, we assume that either $y_{i j}$ or $y_{i j}^{\prime}$ is non-zero for a specific pair of distinct flavors, $i \neq j$, and all other couplings are either exactly zero or negligibly small. For example, if we choose $y_{12}$ to be non-zero, then $y_{i j}=0$ for all $i \neq 1$ and $j \neq 2$, and $y_{i j}^{\prime}=0$ for all $i$ and $j$. The absence of the diagonal coupling is relevant for the suppression of flavor constraints on the model. ${ }^{2}$

The scalar $\varphi$ may also have a self-interaction potential. These interactions are largely irrelevant for this study, with one notable exception: that we assume that $\varphi$ does not acquire a VEV. Such a VEv would shift the Standard Model charged lepton mass eigenstates and introduce mixing. In practice, a violation of the assumption would complicate the model, but for a sufficiently small VEV the salient features of the exotic Higgs decays would be largely preserved and would not appreciably disrupt the behavior of the Higgs. However, the misalignment of mass and Higgs interaction eigenstates could potentially generate sizable contributions to LFV observables.

\footnotetext{
${ }^{2}$ Elements that we set to zero at tree-level are generated at loop-level by the charged lepton violation of the Standard Model, but these processes are suppressed by neutrino masses and are phenomenologically irrelevant to this study.
} 


\subsection{Gauge-invariant effective theory}

Due to the chiral nature of electroweak symmetry, the interactions of (2.1) are not $\mathrm{SU}(2)_{L} \times$ $\mathrm{U}(1)_{Y}$ gauge invariant. Those interactions must be generated by higher-dimension operators that include the Higgs at the electroweak scale. The operators with the lowest possible dimension are

$$
\mathcal{L}_{\varphi-\text { lep }}^{(\mathrm{EW})}=\frac{g_{i j}}{\Lambda} \bar{L}_{i} H E_{j} \varphi+\frac{g_{i j}^{\prime}}{\Lambda} \bar{L}_{i} H E_{j} \varphi^{*}+\text { h.c., } \quad \text { where } y_{i j}^{\left({ }^{\prime}\right)}=\frac{g_{i j}^{\left({ }^{\prime}\right)}}{\Lambda} \frac{v}{\sqrt{2}},
$$

$H$ is the Higgs doublet, $L_{i}$, the lepton doublet of flavor $i$, and $E_{j}$, the lepton singlet of flavor $j$, are expressed in the mass eigenbasis, i.e. the Standard Model Yukawas are diagonal, $\Lambda$ is a mass scale associated with the ultraviolet physics generating that these interactions, and the $g^{(')}$ are dimensionless effective couplings. The low-energy couplings in (2.1) are generated upon inserting the Higgs VEV,$\langle H\rangle=(0, v / \sqrt{2})$. In a case where $y_{i i} \neq 0$, these interactions generate tadpole term for $\varphi$ through the Higgs [54]. Such a tadpole term would destabilize the $\varphi$ potential and necessarily generate a VEV. However, this two-loop tadpole is small enough that it would only minimally impact phenomenology.

Gauge invariance constrains the form of the scalar potential between $\varphi$ and the Higgs:

$$
V_{\varphi-\mathrm{H}}=\kappa H^{\dagger} H \varphi^{*} \varphi+\left(m_{\varphi}^{2}-\kappa \frac{v^{2}}{2}\right) \varphi^{*} \varphi
$$

For the purposes of this study, we may ignore the quartic $\varphi$ self-interactions. For real $\varphi$, there is an additional factor of one-half in (2.3), i.e. $V_{\varphi-\mathrm{H}} \rightarrow V_{\varphi-\mathrm{H}} / 2$.

The underlying dynamics that generate the dimension-5 operators in (2.2) are not relevant for the present study. As a proof of principle, we present three classes of renormalizable theories that generate (2.2) above the electroweak scale in appendix A: vector-like leptons, Froggatt-Nielsen fields, and $R$-parity violating supersymmetry. These do not require any additional light fields, so that it is consistent to examine the Higgs phenomenology of (2.2) independently of a specific ultraviolet completion.

\section{Flavor constraints}

Spurious symmetries are a powerful tool in flavor physics $[55,56]$. When $\varphi$ is complex, the interactions respect a spurious global $L_{i}-L_{j}$ symmetry under which the mediator is charged, $[\varphi]=-2$. This symmetry prohibits many charged lepton-flavor violating tree-level processes [57]. The symmetry is explicitly broken by interactions with the $W$-boson so that higher-order flavor violating processes are suppressed by a loop factor and the ratio of the neutrino and $W$-boson masses; see appendix B. When $\varphi$ is real, there is no such spurious $L_{i}-L_{j}$ symmetry preserved by the interactions. In other words, a real field cannot carry a $\mathrm{U}(1)$ charge.

In this section, we first examine the flavor constraints where only the single, off-diagonal coupling is non-zero. We then explore the flavor constraints in cases with multiple non-zero couplings. 


\subsection{Pure off-diagonal, single coupling}

The limit where the $\varphi$ has only a single off-diagonal flavor coupling is protected from charged lepton-flavor violating processes, insulating the model against most constraints. We briefly review the relevant constraints and refer to section 5.4 of ref. [23] for an indepth discussion.

A mediator with purely LFV interaction can mediate potentially dangerous tree-level charged flavor violation in one notable case: the muonium system, a bound state of an electron and an anti-muon. When $\varphi$ is real and couples to muons and electrons, it may mediate muonium-anti-muonium oscillations [58]. By recasting bounds on $R$-parity violating sneutrinos [59], one obtains a strong bound,

$$
y_{21}, y_{12}<4.4 \times 10^{-4}\left(\frac{m_{\varphi}}{\mathrm{GeV}}\right) \quad[90 \% \mathrm{CL}] \quad(\varphi \text { real }) .
$$

When $\varphi$ is complex, the $L_{\mu}-L_{e}$ spurious symmetry prevents this process at tree-level.

A bound that applies for both real and complex $\varphi$ comes from the interference of $t$-channel $\varphi$ exchange to the forward-backward asymmetry of $e^{+} e^{-} \rightarrow f \bar{f}$ scattering [60],

$$
A_{\mathrm{FB}}^{f}=\frac{\sigma_{>}\left(e^{+} e^{-} \rightarrow f \bar{f}\right)-\sigma_{<}\left(e^{+} e^{-} \rightarrow f \bar{f}\right)}{\sigma_{>}\left(e^{+} e^{-} \rightarrow f \bar{f}\right)+\sigma_{<}\left(e^{+} e^{-} \rightarrow f \bar{f}\right)} .
$$

Unlike the coupling constraints for muonium oscillation, these bounds hold for real or complex $\varphi$. One may recast the $A_{\mathrm{FB}}^{f}$ bounds on sneutrino interactions [61, 62] to the case of a lepton-flavor violating mediator, which yields

$$
y_{21}^{(\prime)}, y_{12}^{(\prime)}<2.5 \times 10^{-3}\left(\frac{m_{\varphi}}{\mathrm{GeV}}\right) \quad y_{31}^{(\prime)}, y_{13}^{(\prime)}<1.1 \times 10^{-3}\left(\frac{m_{\varphi}}{\mathrm{GeV}}\right) \quad[95 \% \mathrm{CL}]
$$

while there are no constraints for $y_{23}^{(\prime)}, y_{32}^{(\prime)}$.

The spurious flavor symmetry also prevents tree-level contributes to rare multi-body lepton decays, $\ell_{i} \rightarrow \ell_{j} \ell_{k} \bar{\ell}_{k}$ and $\ell_{i} \rightarrow \ell_{j} \ell_{k} \bar{\ell}_{k} \nu \bar{\nu}$. When $\varphi$ is real, this process is still prohibited at tree-level because a $\varphi$ emitted by the initial heavy lepton must yield another lepton of the same heavy flavor. In other words, $\varphi$ does not contribute at tree-level to these decays in the absence of a flavor-diagonal coupling to the lighter lepton. Flavor-changing dipole operators in this scenario are down by a loop factor from the Standard Model contribution that is itself suppressed by neutrino masses. As $\varphi$ is hadrophobic, $\mu \rightarrow e$ conversion in the presence of nuclei only occurs at loop-level and, again, requires neutrino masses to violate flavor in the loop.

In summary, the models with a real $\varphi$ with $e$ and $\mu$ are constrained by muonium oscillations (3.1). For either real or complex $\varphi$ interacting with electrons, the primary singlecoupling bound comes from precision measurements of lepton forward-backward asymmetries at lepton colliders (3.3). Otherwise, the single-coupling limit of this model produces negligible signals at traditional charged lepton-flavor violation experiments. There are no appreciable constraints on $\varphi$ coupling with just the $\mu$ and $\tau$. 

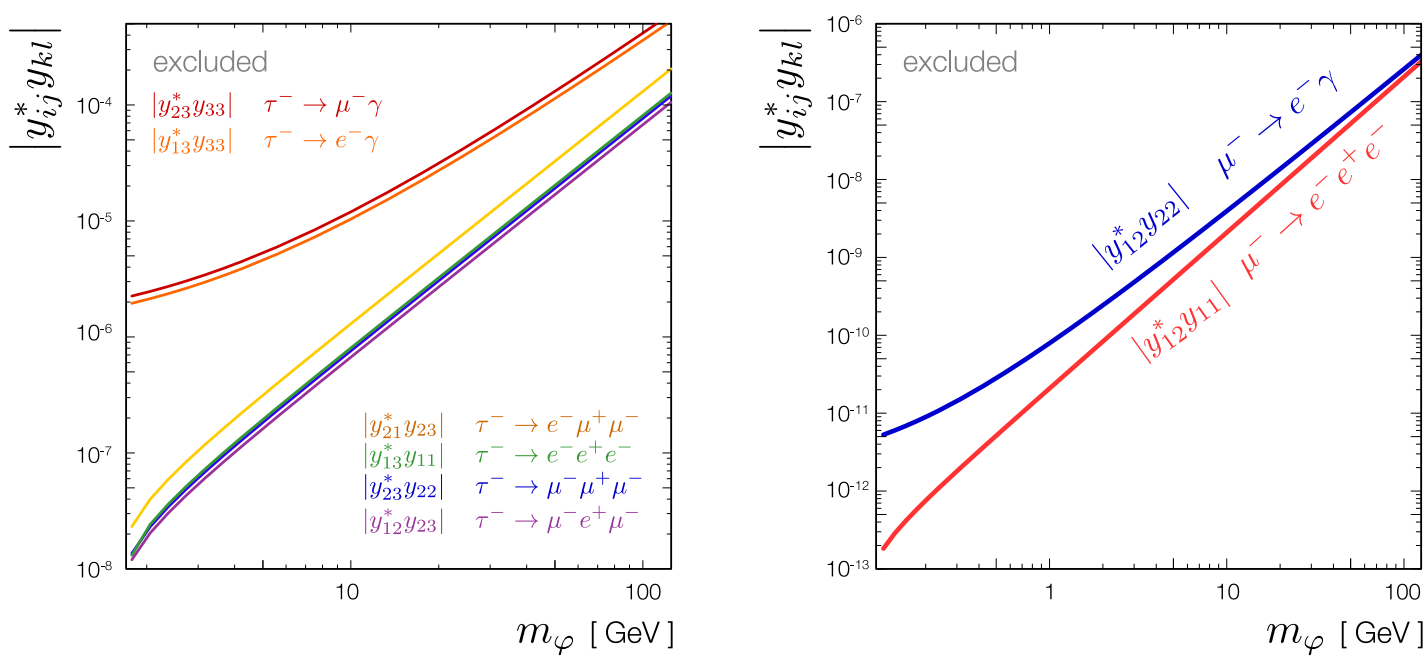

Figure 1. Constraints on the coupling $\left|y_{i j}^{*} y_{k l}\right|$ as a function of mass. Left: LFV constraints from $\tau$ decays. Right: LFV constraints from $\mu$ decays.

\subsection{Multi-coupling}

Section 3.1 shows that precision flavor constraints only moderately impact the single, offdiagonal coupling limit of the $\varphi$. For a more general flavor structure, additional lepton flavor-violating constraints can enter. Given that our scalar is assumed to connect only to the leptonic sector, the primary observables that constrain the model are the exotic decays $\ell_{i} \rightarrow \ell_{j} \gamma$ and $\ell_{i} \rightarrow \ell_{j} \ell_{k} \ell_{l}$. To estimate the effect of relaxing the single-coupling assumption, we examine the effect of turning on an additional coupling.

One of the most promising channels to observe lepton flavor violation is the radiative decays of leptons. The LFV radiative decay branching ratio for a lepton is [63]

$$
\operatorname{Br}\left(\ell_{i} \rightarrow \ell_{j} \gamma\right)=\frac{1}{\Gamma_{i}} \frac{\alpha_{e m} m_{i}^{5}}{64 \pi^{4}}\left(\left|C_{L}\right|^{2}+\left|C_{R}\right|^{2}\right),
$$

where, in this model, the Wilson coefficients can be expressed as

$$
C_{L}=\sum_{k} F\left(m_{i}, m_{k}, m_{j}, m_{\varphi}, y\right) \quad \text { and } \quad C_{R}=\sum_{k} F\left(m_{i}, m_{k}, m_{j}, m_{\varphi}, y^{\dagger}\right)
$$

for the loop function

$$
\begin{array}{rl}
F\left(m_{i}, m_{k}, m_{j}, m_{\varphi}, y\right)=\int_{0}^{1} & d x d y d z \delta(1-x-y-z) \\
& \times \frac{x z m_{j} y_{j k} y_{i k}^{*}+y z m_{i} y_{k j}^{*} y_{k i}+(x+y) m_{k} y_{k j}^{*} y_{i k}^{*}}{4 m_{i}\left(z m_{\varphi}^{2}-x z m_{j}^{2}-y z m_{i}^{2}+(x+y) m_{k}^{2}\right)} .
\end{array}
$$

The measured bounds on these processes for the $\tau$ decays are $\operatorname{Br}(\tau \rightarrow \mu \gamma)<4.4 \times 10^{-8}$ and $\operatorname{Br}(\tau \rightarrow e \gamma)<3.3 \times 10^{-8}$ from BaBar [22], and $\operatorname{Br}(\mu \rightarrow e \gamma)<4.2 \times 10^{-13}$ from the MEG experiment [64]. In the near future, Belle II [65] and MEG-II [66] are expected to improve on these constraints. 
The decay of a lepton into three lighter leptons, $\ell_{i}^{-} \rightarrow \ell_{j}^{-} \ell_{k}^{+} \ell_{l}^{-}$, can be facilitated through tree-level $\varphi$ exchange. The amplitude for these decays can be written

$$
\mathcal{M}_{i j k l}=\frac{\left[\bar{u}\left(p_{i}\right)\left(y_{i j}^{*} P_{L}+y_{j i} P_{R}\right) u\left(p_{j}\right)\right]\left[\bar{v}\left(p_{k}\right)\left(y_{k l}^{*} P_{L}+y_{l k} P_{R}\right) u\left(p_{l}\right)\right]}{m_{k l}^{2}-m_{\varphi}^{2}+i m_{\varphi} \Gamma_{\varphi}}+\delta_{j l}\{j \leftrightarrow l\}
$$

with the invariant masses $m_{i j}^{2} \equiv\left(p_{i}+p_{j}\right)^{2}$. After squaring and spin averaging, the final branching fraction into three leptons can be computed as

$$
\operatorname{Br}\left(\ell_{i}^{-} \rightarrow \ell_{j}^{-} \ell_{k}^{+} \ell_{l}^{-}\right)=\frac{1}{\Gamma_{i}} \int \frac{\left|\mathcal{M}_{i j k l}\right|^{2}}{512 \pi^{3} m_{i}^{3}} d m_{j k}^{2} d m_{k l}^{2} .
$$

Belle places the most stringent constraints on all $\tau^{-} \rightarrow \ell_{j}^{-} \ell_{k}^{+} \ell_{l}^{-}$branching fractions [67], while the $\mu^{-} \rightarrow e^{-} e^{+} e^{-}$bound is from SINDRUM [21]:

$$
\begin{aligned}
& \operatorname{Br}\left(\tau^{-} \rightarrow \mu^{-} \mu^{+} \mu^{-}\right)<2.1 \times 10^{-8}, \quad \operatorname{Br}\left(\tau^{-} \rightarrow \mu^{-} \mu^{+} e^{-}\right)<2.7 \times 10^{-8}, \\
& \operatorname{Br}\left(\tau^{-} \rightarrow \mu^{-} e^{+} \mu^{-}\right)<1.7 \times 10^{-8}, \quad \operatorname{Br}\left(\tau^{-} \rightarrow \mu^{-} e^{+} e^{-}\right)<1.8 \times 10^{-8}, \\
& \operatorname{Br}\left(\tau^{-} \rightarrow e^{-} \mu^{+} e^{-}\right)<1.5 \times 10^{-8}, \quad \operatorname{Br}\left(\tau^{-} \rightarrow e^{-} e^{+} e^{-}\right)<2.7 \times 10^{-8}, \\
& \text { and } \quad \operatorname{Br}\left(\mu^{-} \rightarrow e^{-} e^{+} e^{-}\right)<1.0 \times 10^{-12} \text {. }
\end{aligned}
$$

Belle II [65] and Mu3e [68] are expected to improve these limits considerably.

The most stringent constraint for each combination of two couplings is shown in figure 1. Restricting to two of nine couplings in $y$, allows us to directly bound $\left|y_{i j}^{*} y_{k l}\right| .^{3}$ In comparing these constraints, one should consider that $\left|y_{i j}^{*} y_{k l}\right|$ is constrained here, whereas in the section 3.1, the limits are presented on a single power of $y_{i j}$. Most of the flavor constraints admit a large parameter space that allows for prompt decays. Unsurprisingly, coupling combinations that involve only the first and second generation are more tightly constrained.

\section{Collider}

In this section, we explore the collider sensitivity to the LFV scalar model. We recast a multi-lepton search by the CMS experiment, propose a dedicated search, and comment briefly on the model when $\varphi$ is a long-lived particle.

The model outlined in section 2 allows for two possible exotic Higgs decays into the LFV scalar. The pertinent terms of the Lagrangian are

$$
\mathcal{L} \sim\left(\frac{y_{i j}}{v / \sqrt{2}} \bar{L}_{i} H E_{j} \varphi+\text { h.c. }\right)+\kappa H^{\dagger} H \varphi^{*} \varphi
$$

The first term in (4.1) introduces both the decay, $\varphi \rightarrow \ell_{i}^{+} \ell_{j}^{-}$, and the Higgs decay mode, $h \rightarrow \varphi \ell_{i}^{+} \ell_{j}^{-}$. Despite the minimality of this option, a more compelling pathway comes from

\footnotetext{
${ }^{3}$ If $y_{i i}$ is complex, the contributions in (3.6) depend on the phase of $y_{i i}$. However, it is difficult to induce qualitative changes to limits by modifications to this phase. We present real $y_{i i}$ in expressing the limits.
} 

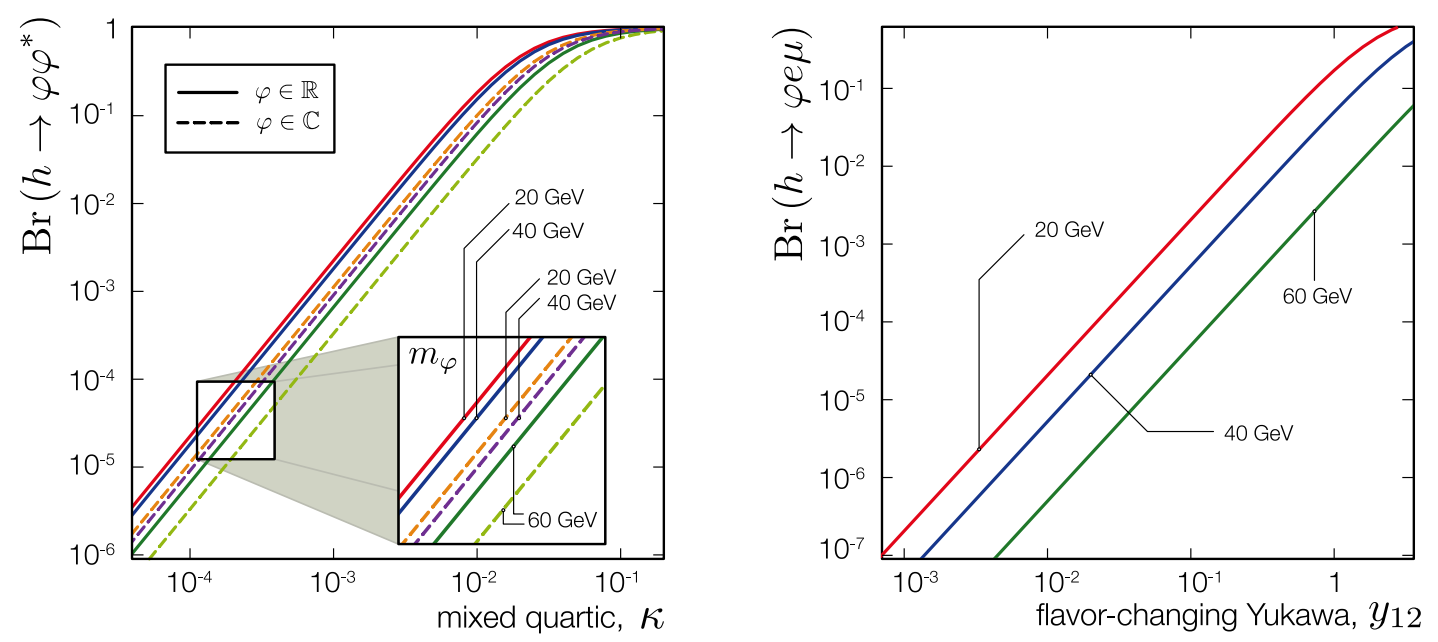

Figure 2. The branching ratio of Left: $h \rightarrow \varphi \varphi^{*}$ and Right: $h \rightarrow \varphi e^{-} \mu^{+}$processes as a function of $\kappa$ (left) and $y_{12}$ (right) for various masses of $\varphi$. The dashed curves are for the cases with real $\varphi$.

the marginal operator of the second term. This generates the $h \rightarrow \varphi \varphi^{*}$ decay. The Higgs decay widths for these processes are

$$
\begin{aligned}
\Gamma_{h \rightarrow \varphi \ell_{i}^{+} \ell_{j}^{-}} & =\frac{y_{i j}^{2}}{128 \pi^{3} m_{h}^{3} v^{2}} \int\left[m_{i j}^{2}-\left(m_{i}+m_{j}\right)^{2}\right] d m_{\varphi i}^{2} d m_{i j}^{2} \\
\Gamma_{h \rightarrow \varphi \varphi^{*}} & =\frac{\kappa^{2} v^{2}}{16 \pi m_{h}} \sqrt{1-\left(\frac{2 m_{\varphi}}{m_{h}}\right)^{2}},
\end{aligned}
$$

where there is an extra factor of $1 / 2$ in the second line for the case with real $\varphi$. Figure 2 shows the branching ratio of the Higgs with Standard Model-like partial width into $h \rightarrow \varphi \varphi^{*}$ and $h \rightarrow \varphi \ell_{i}^{+} \ell_{j}^{-}$as a function of the couplings in (4.1) for three choices of $m_{\varphi}$.

The first term in (4.1) permits the decay of $\varphi$ into two charged leptons of different flavors. When $\varphi$ is complex, the spurious $L_{i}-L_{j}$ symmetry prevents the Higgs decays from producing same-sign lepton pairs, e.g. $\varphi \rightarrow e^{+} \mu^{-}$and $\varphi^{*} \rightarrow e^{-} \mu^{+}$. On the other hand, when $\varphi$ is real, there is no such symmetry and each $\varphi$ decays with equal probability into either sign, e.g. $\varphi \rightarrow e^{+} \mu^{-}$or $e^{-} \mu^{+}$. Thus, a real $\varphi$ means that Higgs decays can yield exotic same-sign lepton pairs.

In the pure off-diagonal, single-coupling case, we explore four different models based on whether $\varphi$ is a real or complex scalar, and on what coupling $y_{12}$ or $y_{23}$ is non-zero. We expect the collider phenomenology of the $y_{13}$ case to be very similar to the $y_{23}$ case, and do not study it in further detail here, but we note that (3.3) bounds $y_{13}$. We list the final states generated for each scenario in table 1. Throughout this section, we assume the off-diagonal Yukawa coupling that enables the $\varphi$ decay is large enough for a prompt signature. This requires $y_{i j} \gtrsim 10^{-6}$. This implies that the new physics generating the dimension-5 operators may not be to far above the PeV scale.

Even when the LFV couplings are small, they could potentially generate an additional LFV signature from $\varphi$-strahlung off of produced lepton pairs. Notably, one could have the 


\begin{tabular}{|llll|}
\hline Model & $\varphi$ & Coupling & Final States \\
\hline $\mathbb{C} e \mu$ & $\mathbb{C}$ & $y_{12} \neq 0$ & $e^{+} e^{-} \mu^{+} \mu^{-}$ \\
$\mathbb{C} \mu \tau$ & $\mathbb{C}$ & $y_{23} \neq 0$ & $\mu^{+} \mu^{-} \tau^{+} \tau^{-}$ \\
$\mathbb{R} e \mu$ & $\mathbb{R}$ & $y_{12} \neq 0$ & $e^{+} e^{+} \mu^{-} \mu^{-}$ \\
$\mathbb{R} \mu \tau$ & $\mathbb{R}$ & $y_{23} \neq 0$ & $\mu^{+} \mu^{+} \tau^{-} \tau^{-}$ \\
\hline
\end{tabular}

Table 1. The four pure off-diagonal, single-coupling models considered in this section. In the cases with real $\varphi$, the opposite-sign cases appear with equal frequency to the more striking same-sign cases listed. We expect $y_{13} \neq 0$ models to have very similar exotic Higgs decay phenomenology to the analogous $y_{23} \neq 0$ model.

decay $Z \rightarrow \varphi \ell_{i}^{+} \ell_{j}^{-}$. Unless $\varphi$ is very light, the $Z$ is the only potentially sizable production pathway beyond Higgs decays. As we will illustrate in section 4.1, $Z$ production is always subdominant to the Higgs pathways.

\subsection{Multi-lepton search limits}

We present the limits from a recasted search for multi-lepton signals by the CMS experiment [53] corresponding to an integrated luminosity of $19.5 \mathrm{fb}^{-1}$ at $\sqrt{s}=8 \mathrm{TeV}$. We consider this specific search as it contains bins with no missing energy requirement, arbitrarily low $S_{T}$, and fairly soft cuts on lepton $p_{T}$. The overwhelming majority of available multi-lepton searches fail to satisfy one or more of these conditions [69-73]. There are also searches for a Higgs decaying into four leptons via two pseudoscalars performed at the Tevatron [74] and the LHC [75-77]. However, in each of those searches the new particle is assumed to decay into same-flavor lepton pairs.

We simulated the production of multi-lepton final states in our model assuming the same event selection criteria as ref. [53]. The model is implemented in FeynRules [78, 79], and we use MadGraph-5 [80] to generate the parton level events. These events are fed into PYTHIA-8 [81] for showering and hadronization using CTEQ6L1 as the parton distribution function and FastJet-3 [82] for jet reconstruction. The program used for the collider analysis is available on GitHub. ${ }^{4}$

We consider four-lepton production through three different processes for each of the models as listed in table 2 (see figure 3 ). In addition to the same-sign process shown, the cases with real $\varphi$ have the opposite-sign final states included when we derive the bounds in figure 4.

A summary of the cuts implemented from ref. [53] are presented in table 3. Lepton identification is modeled using the quoted CMS ID efficiencies in ref. [83] for electrons and muons, and assuming a $70 \%$ efficiency for the hadronic taus [53]. The momenta of leptons are corrected using the CMS detector resolution for electron momentum [84] and assuming a resolution of $1 \%$ in the barrel $(|\eta|<0.9), 3 \%$ in the endcaps $(1.2 \leq|\eta| \leq 2.4)$, and $2 \%$ in the overlap region $(0.9 \leq|\eta|<1.2)$ for muons [85]. The events that pass the cuts are then classified based on the number of leptons $\left(N_{\ell}\right)$, hadronically decaying taus $\left(N_{\tau_{h}}\right)$, and the number of opposite-sign same-flavor leptons $\left(N_{\mathrm{OSSF}}\right)$. The events are further binned

\footnotetext{
${ }^{4}$ https://github.com/ZAKI1905/Pheno
} 


\begin{tabular}{|lllllcr|}
\hline Model & Quartic & \multicolumn{1}{l}{ Process $(p p$ collider $)$} & & Range of $m_{\varphi}$ \\
\hline \multirow{3}{*}{$\mathbb{C} e \mu, \mathbb{C} \mu \tau$} & $\kappa \neq 0$ & $h \rightarrow \varphi+\varphi^{*}$ & $\rightarrow$ & $\ell_{i}^{+}+\ell_{i}^{-}+\ell_{j}^{+}+\ell_{j}^{-}$ & {$[5-60] \mathrm{GeV}$} \\
& $\kappa=0$ & $h \rightarrow \varphi+\ell_{i}^{+}+\ell_{j}^{-}$ & $\rightarrow$ & $\ell_{i}^{+}+\ell_{i}^{-}+\ell_{j}^{+}+\ell_{j}^{-}$ & {$[5-120] \mathrm{GeV}$} \\
& $\kappa=0$ & $Z \rightarrow \varphi+\ell_{i}^{+}+\ell_{j}^{-}$ & $\rightarrow$ & $\ell_{i}^{+}+\ell_{i}^{-}+\ell_{j}^{+}+\ell_{j}^{-}$ & {$[5-90] \mathrm{GeV}$} \\
\hline \multirow{3}{*}{$\mathbb{R} e \mu, \mathbb{R} \mu \tau$} & $\kappa \neq 0$ & $h \rightarrow \varphi+\varphi$ & $\rightarrow$ & $\ell_{i}^{+}+\ell_{i}^{+}+\ell_{j}^{-}+\ell_{j}^{-}$ & {$[5-60] \mathrm{GeV}$} \\
& $\kappa=0$ & $h \rightarrow \varphi+\ell_{i}^{+}+\ell_{j}^{-}$ & $\rightarrow$ & $\ell_{i}^{+}+\ell_{i}^{+}+\ell_{j}^{-}+\ell_{j}^{-}$ & {$[5-120] \mathrm{GeV}$} \\
& $\kappa=0$ & $Z \rightarrow \varphi+\ell_{i}^{+}+\ell_{j}^{-}$ & $\rightarrow$ & $\ell_{i}^{+}+\ell_{i}^{+}+\ell_{j}^{-}+\ell_{j}^{-}$ & {$[5-90] \mathrm{GeV}$} \\
\hline
\end{tabular}

Table 2. The different channels considered in our analysis, along with the ranges of the masses for $\varphi$. For $\kappa=0$, we ignore the interference between the $h$ - and $Z$-mediated channels.
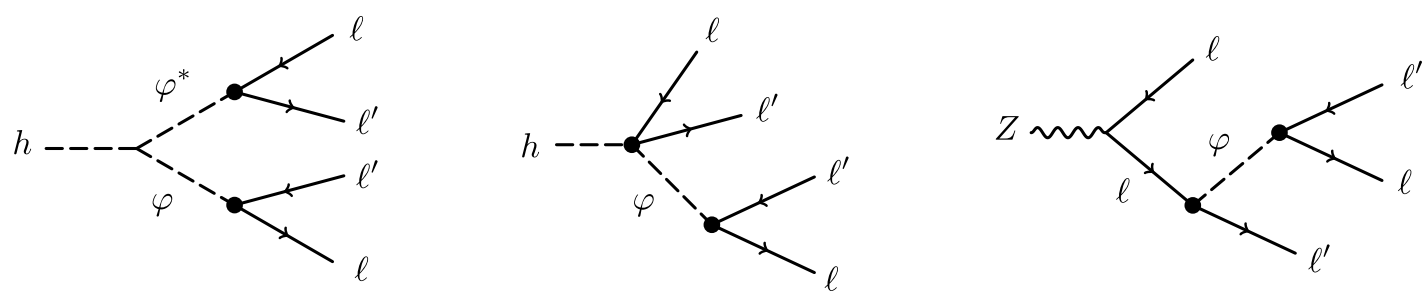

Figure 3. Feynman diagrams for the processes listed in table 2: the dominant diagram for the case with a non-zero quartic coupling $\kappa \neq 0$ (left), the main decay channels involving the Higgs (middle), and $Z$ boson (right) in the case with $\kappa=0$.

according to their $S_{T}$ values, which is the sum of the missing transverse momentum and the scalar sum of the $p_{T}$ of all jets and charged leptons. If there exists an ossf lepton pair for which $75 \mathrm{GeV}<m_{\ell^{+} \ell^{-}}<105 \mathrm{GeV}$, the events are categorized as on- $Z$, otherwise they are labeled off- $Z$. When $N_{\ell}=3$, the off- $Z$ region is additionally divided into $m_{\ell^{+} \ell^{-}}<75 \mathrm{GeV}$ and $105 \mathrm{GeV}<m_{\ell^{+} \ell^{-}}$.

In order to use the CMS results to constrain our models, we apply the following procedure. In each bin, we use a Poisson distribution for $n$ events given an expected rate of $\epsilon \cdot \mathcal{L} \cdot \sigma+B:$

$$
P(n ; \epsilon \cdot \mathcal{L} \cdot \sigma+B)=\frac{e^{-(\epsilon \cdot \mathcal{L} \cdot \sigma+B)}}{n !}(\epsilon \cdot \mathcal{L} \cdot \sigma+B)^{n}
$$

where $\epsilon$ and $B$ are the signal efficiency and the expected background in that specific bin respectively, $\mathcal{L}=19.5 \mathrm{fb}^{-1}$ is the integrated luminosity, and $\sigma$ is the cross-section for the process under consideration. This cross-section can be written as

$$
\sigma=\sigma(p p \rightarrow X) \cdot \operatorname{Br}(X \rightarrow 4 l) \quad X=h, Z
$$

where we use the $8 \mathrm{TeV}$ cross-sections $\sigma(p p \rightarrow h)=24.2 \mathrm{pb}$ [87], $\sigma(p p \rightarrow Z)=34.17 \mathrm{nb}$ [88], and $\mathrm{Br}$ is the branching ratio on which we want to set the limit. The total likelihood is

$$
L=\prod_{i} \frac{e^{-\left(\epsilon_{i} \cdot \mathcal{L} \cdot \sigma+B_{i}\right)}}{n_{i} !}\left(\epsilon_{i} \cdot \mathcal{L} \cdot \sigma+B_{i}\right)^{n_{i}} \cdot P_{\mathrm{LN}}\left(B_{i} \mid \bar{B}_{i}, \delta B_{i}\right),
$$

where the product is over the bins. We model the systematic uncertainty associated with the background prediction as log-normal functions $P_{\mathrm{LN}}\left(B_{i} \mid \bar{B}_{i}, \delta B_{i}\right)$ for the measured value 


\begin{tabular}{|lrl|}
\hline Objects & Conditions & \\
\hline$e^{+} e^{-}, \mu^{+} \mu^{-}$ & $m_{\ell^{+} \ell^{-}}$ & $>12 \mathrm{GeV}$ \\
\hline & $p_{T}^{\text {lead }}$ & $>20 \mathrm{GeV}$ \\
$p_{T}^{\text {sub }}$ & $>10 \mathrm{GeV}$ \\
$|\eta|$ & $<2.4$ \\
& $\sum_{R} E_{T}^{i}$ & $<2 \mathrm{GeV}$ \\
& $p_{T}$ & $>20 \mathrm{GeV}$ \\
& $|\eta|$ & $<2.3$ \\
$\tau_{h}$ & $E_{T}^{i}$ & $<2 \mathrm{GeV}$ \\
& $\sum_{0.1<R\left(\tau_{h}, i\right)<0.3} p_{T}$ & $>30 \mathrm{GeV}$ \\
& $|\eta|$ & $<2.5$ \\
jets & $>R($ jet,$\ell)$ & $>0.3$ \\
&
\end{tabular}

Table 3. Summary of the cuts implemented in our simulation [53]. Jets are reconstructed using the anti-kT algorithm [86] with a distance parameter $R=0.5$, and $\ell$ in the last row stands for the isolated electron, muon, or $\tau_{h}$ candidates.
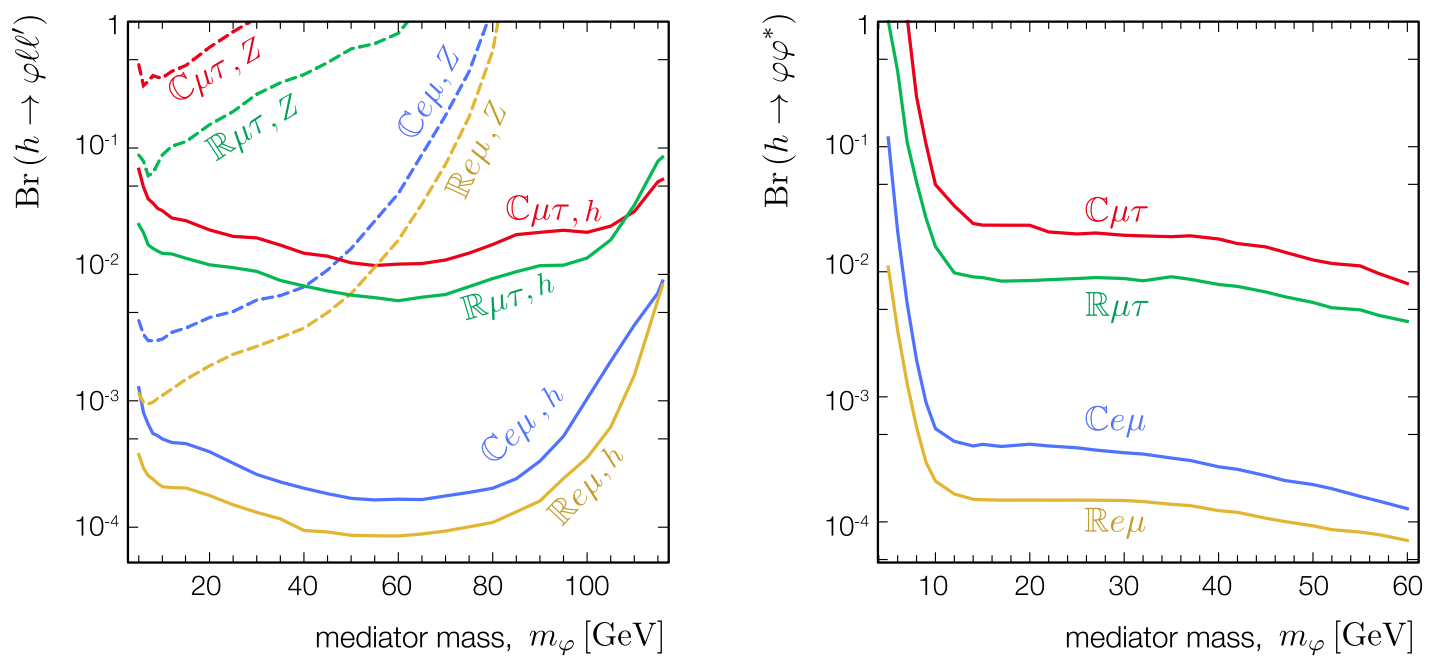

Figure 4. Limits on the branching ratio at $95 \%$ CL for the processes listed in table 2. Left: the limits on the branching ratio from the Higgs-mediated channels (solid) in the case with $\kappa=0$. The limits on $Z$-mediated channels are translated into the equivalent limits on $\operatorname{Br}\left(h \rightarrow \varphi \ell \ell^{\prime}\right)$ using their analytical expressions in (4.8) (dashed). Right: the limits for the case with a non-zero quartic coupling $\kappa \neq 0$.

$B_{i}$, which depends on the expected value $\bar{B}_{i}$ and an uncertainty $\delta B_{i}$. We use the hybrid Bayesian-frequentist approach [89] to marginalize the nuisance parameters by integrating over the background $\left(B_{i}\right)$ errors. We then use this marginal likelihood $\left(L_{m}\right)$ to form the log-likelihood ratio test statistic defined as

$$
Q=-2 \log \left(\frac{L_{m}(s+b)}{L_{m}(b)}\right) .
$$


We calculate limits using the $\mathrm{CL}_{s}[90,91]$ method. We utilize a toy Monte Carlo technique to find the one-sided $p$-value of the observed data in the signal-plus-background and background-only hypotheses, denoted by $p_{s+b}=P\left(Q_{s+b} \geq Q_{\text {obs }}\right)$ and $p_{b}=P\left(Q_{b} \leq Q_{\text {obs }}\right)$ respectively. We define $\mathrm{CL}_{s}$ to be $\mathrm{CL}_{s}=p_{s+b} /\left(1-p_{b}\right)$. We set upper limits on $\mathrm{Br}$ at $95 \%$ confidence by requiring $\mathrm{CL}_{s}<0.05$.

We summarize the limits derived from this method in figure $4 .{ }^{5}$ Bounds on channels with $\tau$ final states are constrained to roughly the level of $\mathrm{Br} \sim 10^{-2}$, while the $e \mu$ channels are constrained to the level of $\mathrm{Br} \sim 10^{-4}$. In the $\tau$ cases, most of the events fall into higher background bins, notably the 3-leptons, $1-\tau_{h}$ and 1-OSSF (0-OSSF) of ref. [53] for Model $\mathbb{C} \mu \tau(\mathbb{R} \mu \tau)$. As one would expect, the real $\varphi$ models $(\mathbb{R} e \mu$ and $\mathbb{R} \mu \tau)$ are more constrained than their complex $\varphi$ counterparts. The limits from $Z$-mediated processes are scaled into the Higgs Br parameter space using:

$$
\operatorname{Br}\left(H \rightarrow \varphi \ell_{1} \ell_{2}\right)=\operatorname{BR}\left(Z \rightarrow \varphi \ell_{1} \ell_{2}\right) \cdot \frac{\Gamma\left(Z \rightarrow \varphi \ell_{1} \ell_{2}\right)}{\Gamma\left(H \rightarrow \varphi \ell_{1} \ell_{2}\right)} \cdot \frac{\Gamma_{h}}{\Gamma_{Z}}
$$

where we have used $\Gamma_{h}=4.07 \mathrm{MeV}$ [87], $\Gamma_{Z}=2.49 \mathrm{GeV}$ [92] as the decay widths of the Higgs and $Z$-boson, respectively.

\subsection{A dedicated Higgs search}

We present a targeted method to probe the parameter space of lepton-flavor violating mediators at the LHC with $\sqrt{s}=13 \mathrm{TeV}$ and a luminosity of $150 \mathrm{fb}^{-1}$. In section 4.1, we showed that the limits from $Z$-boson-mediated diagrams are much weaker than those from the Higgs-mediated processes. As such, we ignore them and focus on the Higgs processes in the rest of this section. We use $\sigma(p p \rightarrow h)=55.1 \mathrm{pb}$ [87] as the $13 \mathrm{TeV}$ production crosssection of the Higgs. Since the Higgs is a resonance, we add a cut on the total invariant mass of the leptons $\left(M_{4}\right)$. This will boost the signal-to-background ratio by eliminating almost all of the background events.

We follow a similar cut procedure as in section 4.1 [53], with the exception that we now relax the $p_{T}^{\text {sub }}$ cut for the sub-leading light leptons to $7 \mathrm{GeV}$ instead of $10 \mathrm{GeV}$. We restrict our study to signatures with four leptons successfully identified at the collider. In $\tau$ systems, we require one hadronically decaying $\tau_{h}$ and one leptonic tau with the different flavor than the other two hard leptons. For example, for non-zero $y_{23}$ coupling we require an electron. We additionally impose a $Z$-window cut on OSSF pairs of $\left|m_{Z}-m_{\ell^{+} \ell^{-}}\right|>15 \mathrm{GeV}$ and apply a final cut on the total invariant mass $\left(M_{4}\right)$ as defined in table 4 .

We can use the number of events passing all of the cuts to place limits on the branching ratios. However, the signal-to-background ratio can be further boosted by binning the events with respect to the lepton pair invariant masses $\left(m_{l_{1}^{+} l_{2}^{-}}, m_{l_{3}^{+} l_{4}^{-}}\right)$in cases with $\kappa \neq 0$ (see figure 3). In model $\mathbb{C} e \mu$, we can record the invariant masses of $e^{+} \mu^{-}, e^{-} \mu^{+}$pairs. For model $\mathbb{R} e \mu$ there is an ambiguity when matching the leptons since there are two ways $e-\mu$ can be paired. We expect the correct combination to have the same invariant masses $\left(m_{\varphi}\right)$ in an ideal measurement. Therefore, from the two possible combinations $(a, b)$ we choose

\footnotetext{
${ }^{5}$ We neglect some of the bins with lower significance to make this procedure computationally tractable.
} 


\begin{tabular}{|rll|}
\hline Model & Required Final States & $M_{4}$ Cut Range $(\mathrm{GeV})$ \\
\hline $\mathbb{C} e \mu$ & $e^{+} e^{-} \mu^{+} \mu^{-}$ & $120-130$ \\
$\mathbb{C} \mu \tau$ & $\tau_{h}^{ \pm} e^{\mp} \mu^{+} \mu^{-}$ & $80-120$ \\
$\mathbb{R} e \mu$ & $e^{+} e^{+} \mu^{-} \mu^{-}$ & $120-130$ \\
$\mathbb{R} \mu \tau$ & $\tau_{h}^{-} e^{-} \mu^{+} \mu^{+}$ & $80-120$ \\
\hline
\end{tabular}

Table 4. The four pure off-diagonal, single-coupling models considered in this section. The opposite-sign final states in model $\mathbb{R} e \mu$ and $\mathbb{R} \mu \tau$ are included in our analysis.
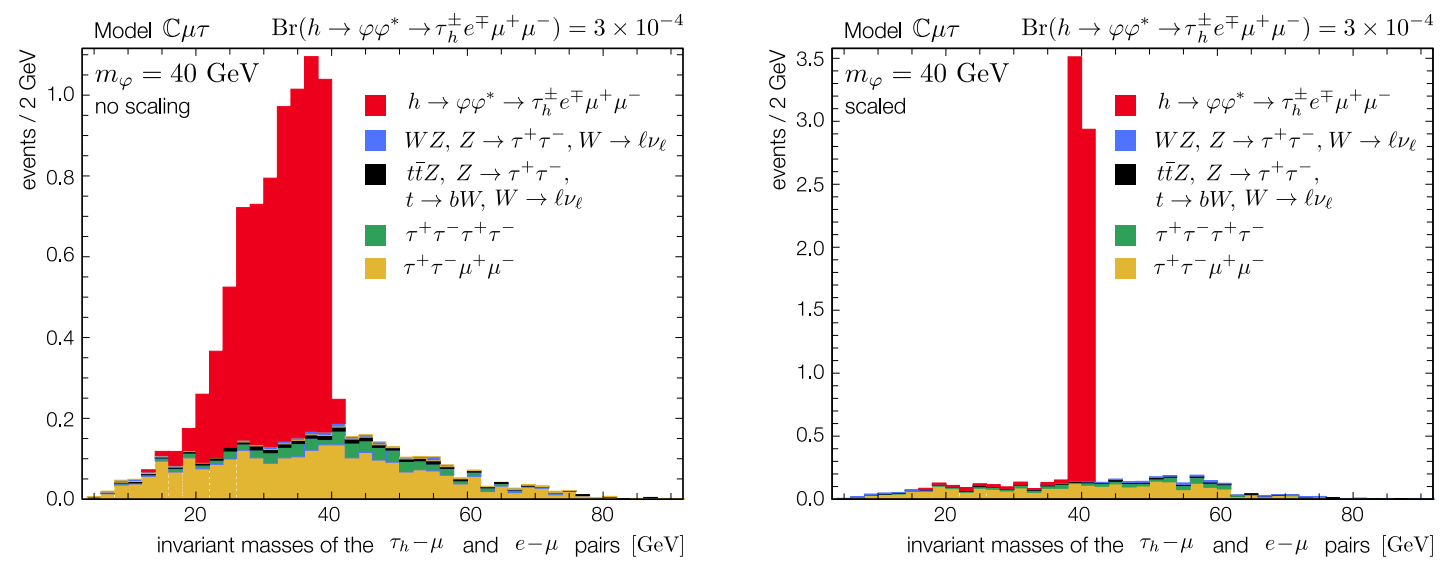

Figure 5. The distribution for invariant masses of $\left(\tau_{h}, \mu\right)$ and $(e, \mu)$ pairs in model $\mathbb{C} \mu \tau$ for the case with $\kappa \neq 0$ for both signal (red) and background channels prior to (left) and after (right) the scaling procedure. In each figure, the mass for both $\left(\tau_{h}, \mu\right)$ and $(e, \mu)$ are shown.

the one with the minimum difference in the invariant masses:

$$
\begin{array}{rlrl}
a: m_{a_{1}}=m_{e_{1}^{+} \mu_{1}^{-}}, & & m_{a_{2}}=m_{e_{2}^{+} \mu_{2}^{-}}, & \Delta m_{a}=\left|m_{a_{2}}-m_{a_{1}}\right| \\
b: m_{b_{1}}=m_{e_{2}^{+} \mu_{1}^{-}}, & & m_{b_{2}}=m_{e_{1}^{+} \mu_{2}^{-}}, & \Delta m_{b}=\left|m_{b_{2}}-m_{b_{1}}\right| \\
\Delta m & \longrightarrow \quad \operatorname{Min}\left(\Delta m_{a}, \Delta m_{b}\right) . &
\end{array}
$$

In model $\mathbb{C} \mu \tau$, the invariant masses of the lepton pairs are less than $m_{\varphi}$ due to the missing energy in $\tau$ decays. In order to approximately correct for this missing energy, we perform a momentum scaling of the decay products as described below. Assuming that the $\tau$ s are highly boosted, the missing and visible momenta from the $\tau$ decay $\left(\mathbf{p}_{\tau}^{\text {miss }}\right.$ and $\mathbf{p}_{\tau}^{\text {vis }}$, respectively) are approximately collinear. Therefore, the true $\tau$ momentum can be written as $p_{\tau}=p_{\tau}^{\text {miss }}+p_{\tau}^{\text {vis }}=\alpha p_{\tau}^{\text {vis }}$, where $\alpha$ is a scaling constant that we wish to determine. This visible momentum $\left(p_{\tau}^{\mathrm{vis}}\right)$ is equal to the electron's momentum $\left(p_{e}\right)$ in the case of leptonic $\tau$ decays, i.e. $\tau \rightarrow e \bar{\nu}_{e} \nu_{\tau}$, so we implement a scaling of the $\tau_{h}^{ \pm}, e^{\mp}$ four-momenta:

$$
\begin{array}{rlrl}
p_{\tau_{h}}^{\mathrm{vis}} \longrightarrow p_{\tau_{h}} & =\alpha_{\tau_{h}} p_{\tau_{h}}^{\mathrm{vis}} & \alpha_{\tau_{h}} & =\left(\frac{m_{X}}{m_{\tau_{h} \mu}}\right)^{2} \\
p_{\tau_{\ell}}^{\mathrm{vis}}=p_{e} \longrightarrow p_{\tau_{\ell}}=\alpha_{e} p_{e} & \alpha_{e}=\left(\frac{m_{X}}{m_{e \mu}}\right)^{2} .
\end{array}
$$


We can determine $m_{X}$, which can be identified with $m_{\varphi}$, by imposing the total invariant mass constraint,

$$
\operatorname{Inv}\left(e^{\prime}, \mu, \tau_{h}^{\prime}, \mu\right) \approx m_{h}=125 \mathrm{GeV},
$$

where the four-momenta for $e^{\prime}$ and $\tau_{h}^{\prime}$ are scaled as in (4.10). After solving (4.12) for $m$ and plugging it back into (4.10), we derive the scaled momenta and use them for binning the events. This scaling is justified because in the relativistic limit for the $e-\mu$ pair, we have $m_{e \mu} \approx \sqrt{2 p_{e} \cdot p_{\mu}}$. A scaling of $p_{e}$ by $\alpha_{e}$ results in a scaling $\sqrt{\alpha_{e}}$ in $m_{e \mu}$. As for the $\tau_{h}-\mu$ pair, the ratio of the scaled invariant mass $\left(p_{\tau_{h}}+p_{\mu}\right)^{2}=m_{\tau_{h} \mu}^{\prime 2}$ to the unscaled invariant mass $\left(p_{\tau_{h}}^{\mathrm{vis}}+p_{\mu}\right)^{2}=m_{\tau_{h} \mu}^{2}$ is:

$$
\left(\frac{m_{\tau_{h} \mu}^{\prime}}{m_{\tau_{h} \mu}}\right)^{2} \approx \frac{\alpha_{\tau_{h}}^{2} m_{\tau_{h}}^{2}+2 \alpha_{\tau_{h}} p_{\tau_{h}} \cdot p_{\mu}}{m_{\tau_{h} \mu}^{2}} \approx \alpha_{\tau_{h}}+\left(\frac{m_{\tau_{h}}}{m_{\tau_{h} \mu}}\right)^{2} \alpha_{\tau_{h}}\left(\alpha_{\tau_{h}}-1\right) .
$$

If we take $m_{\tau_{h} \mu} \approx 10 \mathrm{GeV}$ and also assume that the scaling factor is close to one, the sub-leading terms in (4.13) are much less than $10^{-3}$. The result of this scaling for a sample case $\left(m_{\varphi}=40 \mathrm{GeV}\right)$ is shown in figure 5. This scaling method is exceptional at sharpening the signal without inducing any spurious focusing of the background distribution.

In model $\mathbb{R} \mu \tau$, due to the missing energies we can no longer resolve the ambiguity in matching the leptons by using the invariant masses, so we simply use the number of events passing all the cuts to set limits in this case. However, the scaling procedure outlined above can still be performed to find the location of a bump, i.e. $m_{\varphi}$, in the event of an excess. The scaling should be done for both possible combinations of $\left(\tau_{h}-\mu, e-\mu\right)$ pairs. The solutions to (4.12), $\alpha_{\mu, \tau}^{1,2}$, must be checked. If either $\alpha_{\tau_{h}}$ or $\alpha_{e}$ in a combination is less than one, the combination is incorrect and is discarded. The cases with more than one correct scaling combination are ambiguous, but the $m_{\varphi}$ feature should still emerge clearly. Alternatively, one could use only the unambiguous set of scaled invariant masses to determine the mediator mass.

The scaling method works well for $h \rightarrow \varphi \varphi^{*}$, but it is not able to enhance sensitivity to the $h \rightarrow \varphi \ell_{i}^{+} \ell_{j}^{-}$signals as there is only a single resonance. However, as can be observed on the left side of figure 5 , the distribution does create a kinematic endpoint in the invariant mass which could be used to ascertain the internal resonance mass.

We consider three background channels for model $\mathbb{C} e \mu$ and $\mathbb{R} e \mu$ : (a) $p p \rightarrow e^{+} e^{-} \mu^{+} \mu^{-}$, (b) $t \bar{t} Z$, (c) $W^{+} W^{-} Z$, where $t \rightarrow W b$, and $W / Z$ bosons decay leptonically. The dominant background in model $\mathbb{C} e \mu$ is from $p p \rightarrow e^{+} e^{-} \mu^{+} \mu^{-}$, while the 2-Sssf background in model $\mathbb{R} e \mu$ is negligible. For model $\mathbb{C} \mu \tau$ and $\mathbb{R} \mu \tau$, we analyzed the background from (a) $p p \rightarrow$ $\mu^{+} \mu^{-} \tau^{+} \tau^{-}$, (b) $\tau^{+} \tau^{-} \tau^{+} \tau^{-}$, (c) $t \bar{t} Z$, (d) $W^{+} W^{-} Z$ processes. The background originating from jets misidentified as $\tau_{h}$ ("fake taus") in three main channels (a) $t \bar{t} W$, (b) $W Z$, (c) $t \bar{t} Z$ are also included. In order to estimate this fake background, we first generate events with $n$ jets +3 light leptons final states with FastJet-3 [82] using anti-kT algorithm [86] with a distance parameter $R=0.4$. We then form a set of $n$ events corresponding to each jet, which are weighted using the jet $\rightarrow \tau_{h}$ misidentification rate [93], which is typically of $\mathcal{O}(1 \%)$. Finally, we normalize the background events in all cases by

$$
w=\frac{\mathcal{L} \cdot \sigma}{N} \cdot K
$$




\begin{tabular}{|lll|}
\hline Model & Process & Signal Region $(\mathrm{GeV})$ \\
\hline $\mathbb{C} e \mu$ & $h \rightarrow \varphi \varphi^{*}$ & $m_{e^{+} \mu^{-}}$and $m_{e^{-} \mu^{+}} \in\left[m_{\varphi}-3, m_{\varphi}+3\right]$ \\
$\mathbb{C} e \mu$ & $h \rightarrow \varphi e^{+} \mu^{-}$ & $m_{e^{+} \mu^{-}}$or $m_{e^{-} \mu^{+}} \in\left[m_{\varphi}-3, m_{\varphi}+3\right]$ \\
$\mathbb{C} \mu \tau$ & $h \rightarrow \varphi \varphi^{*}$ & $m_{\tau_{h}^{ \pm} \mu^{\mp}}^{\prime}$ and $m_{e^{\mp} \mu^{ \pm}}^{\prime} \in\left[m_{\varphi}-5, m_{\varphi}+5\right]$ \\
$\mathbb{C} \mu \tau$ & $h \rightarrow \varphi \tau^{+} \mu^{-}$ & All values of $m_{\tau_{h}^{+} \mu^{\mp}}$ and $m_{e^{\mp} \mu^{ \pm}}$ \\
$\mathbb{R} e \mu$ & $h \rightarrow \varphi \varphi^{*}$ & $m_{e^{+} \mu^{-}}$and $m_{e^{+} \mu^{-}} \in\left[m_{\varphi}-3, m_{\varphi}+3\right]$ \\
$\mathbb{R} e \mu$ & $h \rightarrow \varphi e^{+} \mu^{-}$ & All values of $m_{e^{+} \mu^{-}}$and $m_{e^{+} \mu^{-}}$ \\
$\mathbb{R} \mu \tau$ & Both & All values of $m_{\mu^{+} \tau_{h}^{-}}$and $m_{\mu^{+} e^{-}}$ \\
\hline
\end{tabular}

Table 5. Definition of signal bins used to place limits on the branching ratios in each case. For the OsSF events in model $\mathbb{R} e \mu$ and $\mathbb{R} \mu \tau$, we use the corresponding definition from model $\mathbb{C} e \mu$ and $\mathbb{C} \mu \tau$.

where $\sigma$ is the production cross-section for each specific background channel at $\sqrt{s}=13$ $\mathrm{TeV}$ and we take $\mathcal{L}=150 \mathrm{fb}^{-1}$. We also assume the $\mathrm{K}$-factor $\sim 1.7$ [94] to correct for the NNLO effects. The distribution of invariant masses of $\tau_{h}-\mu$ and $e-\mu$ pairs (for both signal and background) with an example choice of parameters in model $\mathbb{C} \mu \tau$ are shown in figure 5. It can be seen that the cut procedure eliminates most of the background events in this case, and similarly in other models.

We follow the same statistical methods to set limits on the branching ratios, with a different binning procedure. We used one bin for models with OSSF signatures, and two bins for model $\mathbb{R} e \mu$ and $\mathbb{R} \mu \tau$, i.e. for the OSSF and SSSF contributions. The definition of these signal bins is presented in table 5 .

In figure 6, we summarize the projected limits from our search proposal, reproduce the CMS search at $\sqrt{s}=8 \mathrm{TeV}$ [53] (section 4.1), and add to these the same CMS limits naïvely projected to $\sqrt{s}=13 \mathrm{TeV}$ and $150 \mathrm{fb}^{-1}$. These projected CMS bounds are estimated by

$$
\mathrm{BR}_{13}=\mathrm{BR}_{8} \cdot \sqrt{\frac{19.5 \mathrm{fb}^{-1}}{150 \mathrm{fb}^{-1}}} \cdot \frac{\sigma_{8}(p p \rightarrow h)}{\sigma_{13}(p p \rightarrow h)}
$$

where $\sigma_{13}(p p \rightarrow h)=55.1 \mathrm{pb}[87]$, and $\sigma_{8}(p p \rightarrow h)=24.2 \mathrm{pb}[87]$.

\subsection{Long-lived LFV scalars}

Throughout this work, we have assumed that $\varphi$ decays promptly in the detector. In principle, it could be long-lived and yield a displaced signature in the detector. The lifetime of $\varphi$ is

$$
\Gamma\left(\varphi \rightarrow \ell_{i}^{+} \ell_{j}^{-}\right)=\frac{m_{\varphi}}{16 \pi}\left[\left(\left|y_{i j}\right|^{2}+\left|y_{i j}^{\prime}\right|^{2}\right)\left(1-r_{i}^{2}-r_{j}^{2}\right)-4 r_{i} r_{j} \operatorname{Re}\left(y_{i j}^{*} y_{i j}^{\prime}\right)\right] \lambda^{1 / 2}\left(1, r_{i}^{2}, r_{j}^{2}\right),
$$

where $r_{i}=m_{\ell_{i}} / m_{\varphi}$ and $\lambda(a, b, c)=a^{2}+b^{2}+c^{2}-2(a b+b c+a c)$ is the phase space factor. Assuming a single, off-diagonal coupling $y_{i j} \neq 0$, the characteristic displacement scale is

$$
c \tau_{\varphi} \approx 500 \mu \mathrm{m}\left(\frac{10 \mathrm{GeV}}{m_{\varphi}}\right)\left(\frac{10^{-6}}{\left|y_{i j}\right|}\right)^{2} \frac{\lambda^{-1 / 2}\left(1, r_{i}^{2}, r_{j}^{2}\right)}{1-r_{i}^{2}-r_{j}^{2}} .
$$



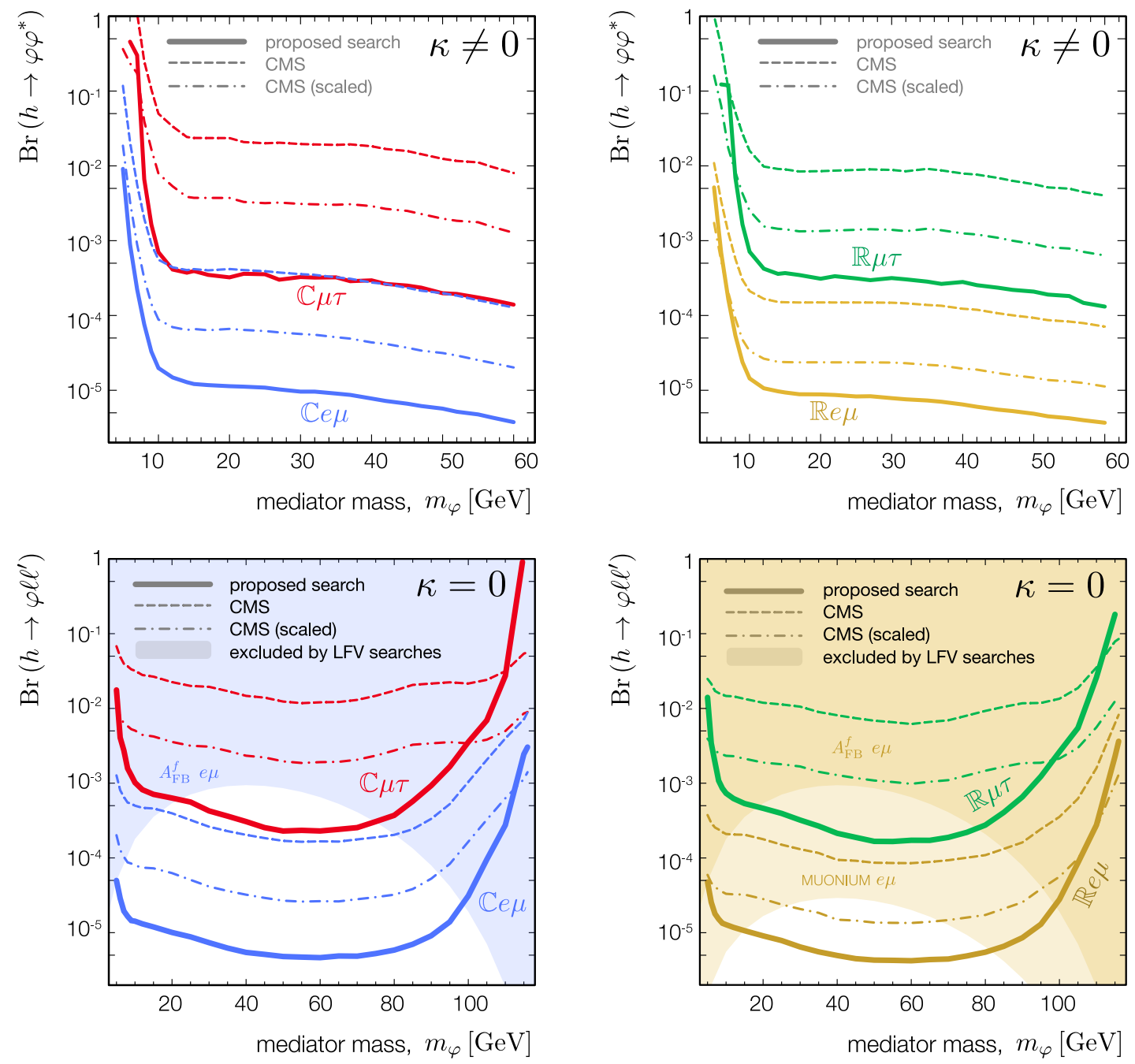

Figure 6. Limits on the branching ratio at 95\% CL for the models listed in table 1 in the case with a non-zero quartic coupling $\kappa \neq 0$ (top), and a zero quartic coupling $\kappa=0$ (bottom). The dashed curves are CMS results at $8 \mathrm{TeV}$ [53]. The dot-dashed curves are the same results scaled using (4.15). The thick curves correspond to our search proposal at $\sqrt{s}=13 \mathrm{TeV}$, and a luminosity of $150 \mathrm{fb}^{-1}$. The bottom two figures show the excluded region from the most relevant lepton-flavor violating searches in the $e \mu$ cases. The $\mu \tau$ cases are unconstrained.

Targeted searches are typically much more sensitive to long-lived particles than their prompt counterparts. This is especially true with leptons, as impact parameter criteria designed to remove cosmic muons, material interactions, and other rare backgrounds run the danger of removing these non-prompt leptons from the signal entirely [95, 96]. Many LHC searches exist for long-lived particles. Of those in the lepton channels, the $p_{T}$ requirements at the trigger level can be quite harsh, removing sensitivity to Higgs decays, e.g. [97-99], others are focused on displaced $e e$ or $\mu \mu$ signatures, e.g. [100, 101], and others target fairly specific signatures [102]. Recasting these studies is beyond the scope of this work, but we note that scalars with an LFV decay in these models can easily be displaced and are not optimally constrained by existing searches. 


\section{Conclusion}

Exotic Higgs decays are one of the most promising places to uncover new physics in the near future. In order to ensure no signals gets overlooked, it is essential to have a comprehensive program. In this work, we explored a simple model containing a new scalar lighter in mass than the Higgs that decays into two standard model charged leptons of different flavor. Although there is substantial motivation to consider new physics within the lepton sector from many extant anomalies, this specific signature had been overlooked thus far at the LHC.

At the weak scale, the LFV decay of the new scalar originates from dimension-5 operators involving the Higgs, which also facilitates a decay path for the Higgs $h \rightarrow \varphi \ell \ell^{\prime}$. The operator can originate from a variety of simple ultraviolet completions. The addition of a dimension-4 coupling allows the direct decay $h \rightarrow \varphi \varphi^{*}$. In the case of a real scalar, this can result in an exotic signature with two same-sign same-flavor pairs of leptons, e.g. $e^{+} e^{+} \mu^{-} \mu^{-}$.

New LFV couplings can impact many precision and flavor observables. We show that constraints from these prove very mild if the flavorful couplings to leptons are completely off-diagonal, with the most stringent being forward-backward asymmetries for couplings involving electrons, and muonium oscillations for a real scalar with $y_{12} \neq 0$. In the case where the alignment is not exact, additional observables can be constraining, notably lepton radiative decays and decays into three charged leptons. Prompt scalar decays can very easily be accommodated within these constraints.

An existing low missing energy and low $S_{T}$ multi-lepton search can be used to place constraints on this model, but a dedicated search that capitalizes on the distinctive kinematics can perform much better. In such a search, requiring a four-lepton invariant mass consistent with a Higgs parent allows for a rejection of most of the small backgrounds. Even in the cases with taus, information can be gleaned about the mass of the new scalar through application of kinematic end points or by enforcing a Higgs mass constraint. While prompt signatures were the focus of this work, the new scalars could instead be displaced. Such a striking displaced signal with relatively soft leptons could potentially benefit from a dedicated search.

\section{Acknowledgments}

We thank J. Brod, I. Galon, K.C. Kong, D. McKeen, J. Shelton, and J. Zupan for useful comments and discussions. We also thank Y.C. Ding for his assistance with technology. J.A.E. and P.T. thank the Aspen Center for Physics (NSF PHY-1066293) for its hospitality when this work was initiated. P.T. thanks the Kavli Institute for Theoretical Physics (NSF PHY-1748958) for its hospitality while part of this manuscript was being completed. J.A.E. acknowledges support by DOE grant DE-SC0011784. P.T. is supported by the DOE grant de-sc0008541. M.Z. is supported by the CAS PIFI grant \#2019PM0110. 


\section{A Renormalizable ultraviolet completions}

The dimension-5 weak-scale effective operator (2.2) can emerge from renormalizable theories whose additional degrees of freedom have been integrated out. We present three simple example models that do not introduce additional low-energy states that would influence the phenomenology.

\section{A.1 Vector-like leptons}

One of the simplest extensions is to introduce a vector-like pair of new leptons, $\xi_{L, R}$,

$$
\mathcal{L}_{\xi}^{V} \supset M \bar{\xi}_{L} \xi_{R}+\left[\lambda_{i} \bar{L}_{i} \cdot H \xi_{R}+\lambda_{i}^{\prime} \bar{\xi}_{L} E_{i} \varphi+\text { h.c. }\right] .
$$

We have chosen the electroweak quantum numbers of $\xi_{L, R}$ such that $\xi_{R}$ and $E$ have the same charges. Because the new fermions form a vector-like pair, the Dirac mass $M$ may be naturally large. The $y_{i j}$ in (2.1) are generated by a tree-level diagram with virtual heavy leptons. In turn, the low-energy effective interactions in (2.1) appear upon inserting the Higgs vacuum expectation value. For example,

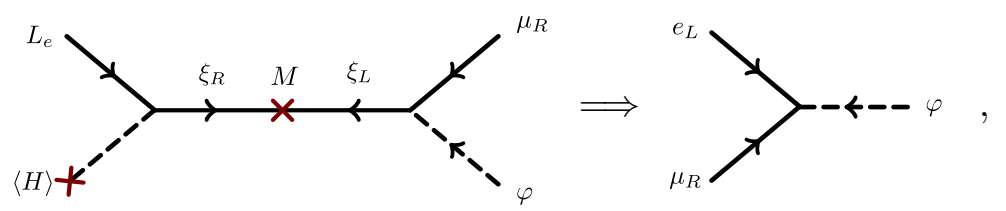

where fermion arrows indicate helicity and the $\varphi$ arrow indicates $L_{e}-L_{\mu}$ charge. The effective scale in (2.2) is then identified with the mass of the heavy fermion, $\Lambda \sim M$. Ref. [54] recently explored the loop-level implications of this class of ultraviolet completion. In this model, the off-diagonal nature is not manifestly enforced. Essentially, one needs to introduce a $\lambda$ and $\lambda^{\prime}$ that are differently flavor directed and well aligned with the mass eigenbasis in flavor space, e.g. $\lambda \sim(1,0,0)$ and $\lambda^{\prime} \sim(0,1,0)$.

\section{A.2 Froggatt-Nielsen}

The Froggatt-Nielsen mechanism generates the Standard Model fermion mass hierarchy by integrating out heavy degrees of freedom that break Abelian flavor symmetries [103]. We may apply this framework with a single $\mathrm{U}(1)_{X}$ flavor symmetry and heavy scalar, $S$,

$$
\mathcal{L}_{\text {Lep. }}^{\mathrm{FN}} \supset \alpha_{i j} \bar{L}_{i} \cdot H E_{j}\left(\frac{S}{M}\right)^{\left|n_{i j}^{Y}\right|} .
$$

The scalar field $S$ has $\mathrm{U}(1)_{X}$ flavor charge $[S]_{X}=-1$ so that the power $n_{i j}^{Y}=\left[\bar{L}_{i}\right]_{X}+$ $[H]_{X}+\left[E_{j}\right]_{X}$. We assume $\alpha \sim \mathcal{O}(1)$, and $M$ is the scale at which the heavy degrees of freedom were integrated out. Assume that $\mathrm{U}(1)_{X}$ is broken at roughly the scale $M$ by $\langle S\rangle \approx 0.2 M$. This generates the Standard Model Yukawa hierarchy for the charged leptons. The Lagrangian in (2.2) can be generated similarly, as

$$
\mathcal{L}_{\varphi \text {-lep. }}^{\mathrm{FN}} \supset \beta_{i j} \bar{L}_{i} \cdot H E_{j}\left(\frac{S}{M}\right)^{\left|n_{i j}^{g}\right|} \frac{\varphi}{\Lambda}+\beta_{i j}^{\prime} \bar{L}_{i} \cdot H E_{j}\left(\frac{S}{M}\right)^{\mid n_{i j}^{g^{\prime} \mid}} \frac{\varphi^{*}}{\Lambda},
$$


where $n_{i j}^{g}=\left[\bar{L}_{i}\right]_{X}+[H]_{X}+\left[E_{j}\right]_{X}+[\varphi]_{X}$. For a review of the Froggatt-Nielsen mechanism, and a more phenomenologically realistic example, see ref. [23], where a product of two $\mathrm{U}(1)$ flavor symmetries is implemented.

\section{A.3 R-parity violating supersymmetry}

Finally, one may directly generate (2.1) without the intermediate step (2.2) if one identifies the mediator $\varphi$ as a sneutrino in $R$-parity violating supersymmetry [104-106]:

$$
W_{R_{p}} \supset \frac{1}{2} \sum_{i, j, k} \lambda_{i j k} \mathbb{L}_{i} \cdot \mathbb{L}_{j} \cdot \overline{\mathbb{E}}_{k}
$$

where $i, j, k$ are generation indices, $\mathbb{L}$ is the lepton $\mathrm{SU}(2)_{L}$ doublet superfield, and $\overline{\mathbb{E}}$ is the electron singlet superfield. Electroweak and flavor symmetries require this term to be anti-symmetric in $\{i, j\}$, i.e. $i \neq j$. If we extract the Yukawa couplings from this term we get

$$
\mathcal{L}_{L L \bar{E}}=\lambda_{i j k}\left[\widetilde{\nu}_{L}^{i} \bar{e}_{R}^{k} e_{L}^{j}+\widetilde{e}_{L}^{j} \bar{e}_{R}^{k} \nu_{L}^{i}+\left(\widetilde{e}_{R}^{k}\right)^{*}\left(\bar{\nu}_{L}^{i}\right)^{c} e_{L}^{j}-(i \leftrightarrow j)\right]+\text { h.c. },
$$

in which we can identify the mediator $\varphi$ with the sneutrino $\widetilde{\nu}_{L}$. In this case, the interactions with the $\varphi$ and the Higgs in (2.3) are generated through $D$-terms and soft terms $[107$, eq. (2.5)].

However, this framework suffers from two major problems. First, it is challenging to decouple the slepton portion of the doublet from the sneutrino. A light charged slepton is fairly difficult to conceal from a variety of searches. Second, the sneutrino itself can be amply produced through a $Z$ boson, and, if $m_{\widetilde{\nu}}<m_{Z} / 2$ would appreciably correct the $Z$ width. Fortunately, a fairly simple resolution would be to introduce a right-handed neutrino superfield $\mathbb{N}$. A small $A$-term $H \widetilde{N} \widetilde{L}$ would generate a slight right-handed lefthanded sneutrino mixing. This light right-handed sneutrino is a viable $\varphi$ candidate. While an interesting possibility, further exploration of this model is well beyond the scope of this work.

\section{B Review of chiral structure}

For clarity, we will briefly review the chiral structure of the Yukawa interaction that plays an important role in our model. For more details than presented here, see, Martin's 2011 TASI lectures [108] or the comprehensive version with Dreiner and Haber [109]. A Dirac fermion $\Psi$ is a mixture of left-handed $(\psi)$ and right-handed $(\bar{\chi})$ Weyl fermions with the same conserved charges, which can be represented in the Dirac basis for the $\gamma$-matrices as

$$
\Psi=\left(\begin{array}{c}
\psi \\
\bar{\chi}
\end{array}\right) \quad \bar{\Psi} \equiv \Psi^{\dagger} \gamma^{0}=(\chi \bar{\psi}) .
$$

In this notation, barred (unbarred) Weyl spinors are understood to be right-(left-)handed. Complex conjugation converts a left-chiral fermion into a right-chiral anti-fermion, so one 

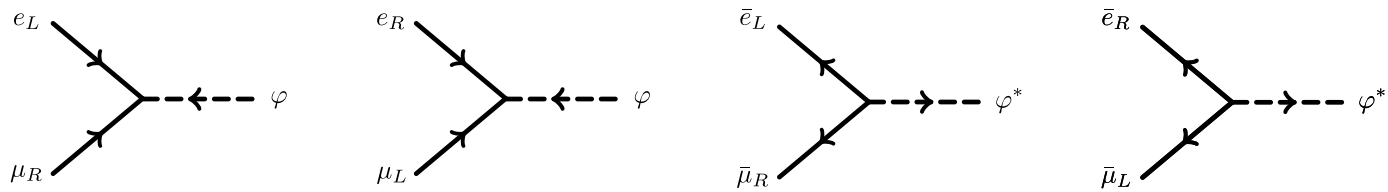

Figure 7. Vertices from (B.3) to those in (B.4) where arrows on fermions represent helicity and the arrow on the scalar represents $L_{e}-L_{\mu}$ charge.

may consider $\bar{\chi}=\chi^{\dagger}$ where $\chi$ is the left-handed CP conjugate of $\bar{\chi}$. Note that left- and rightchiral spinors have different indices ${ }^{6}$ that cannot be contracted with one another as they are in different induced representations of the Poincarè group. We explicitly decompose a Dirac electron field, $e$, into its Weyl components $e_{L}$ and $e_{R}$ :

$$
e=\left(\begin{array}{c}
e_{L} \\
\bar{e}_{R}
\end{array}\right) \quad \bar{e}=\left(\begin{array}{ll}
e_{R} & \left.\bar{e}_{L}\right) .
\end{array}\right.
$$

The subscripts $L, R$ differentiate two fundamentally different fields in the Standard Model.

- $e_{L}$ is a left-handed electron with charge $Q=-1$ that is part of an electroweak doublet.

- $\bar{e}_{L}$ is its conjugate, a right-handed positron $(Q=+1)$ that is part of an electroweak doublet.

- $\bar{e}_{R}$ is a right-handed electron with charge $Q=-1$ that is an electroweak singlet.

- $e_{R}$ is its conjugate, a left-handed positron $(Q=+1)$ that is an electroweak singlet.

The Dirac muon field $\mu$ can be analogously decomposed into Weyl $\mu_{L, R}$ and $\bar{\mu}_{L, R}$ fields. With respect to these Weyl fermions, the interactions in (2.1) are

$$
\begin{aligned}
y_{12} \bar{e} P_{L} \mu \varphi & =y_{12} e_{R} \mu_{L} \varphi & y_{12}^{\prime} \bar{e} P_{R} \mu \varphi & =y_{12}^{\prime} \bar{e}_{L} \bar{\mu}_{R} \varphi \\
\left(y_{12} \bar{e} P_{L} \mu \varphi\right)^{*} & =y_{12}^{*} \bar{e}_{R} \bar{\mu}_{L} \varphi^{*} & \left(y_{12}^{\prime} \bar{e} P_{R} \mu \varphi\right)^{*} & =\left(y_{12}^{\prime}\right)^{*} e_{L} \mu_{R} \varphi^{*} .
\end{aligned}
$$

These are four distinct interactions, though the reality of the Lagrangian connects the terms in (B.3) to those in (B.4). These interactions are shown diagrammatically in figure 7, where arrows correspond to fermion helicity. To aid in translation between the four-component and two-component notation, we write (2.1) in terms of the Weyl fields:

$$
\mathcal{L} \supset\left(y_{i j} \ell_{R i} \ell_{L j} \varphi+y_{i j}^{*} \bar{\ell}_{R i} \bar{\ell}_{L j} \varphi^{*}\right)+\left(y_{i j}^{\prime} \bar{\ell}_{L i} \bar{\ell}_{R j} \varphi+y_{i j}^{*} \ell_{L i} \bar{\ell}_{R j} \varphi^{*}\right)
$$

An example where this formalism is useful is to examine possible loop-level contributions to flavor-changing dipole operators. We can see that such operators necessarily

\footnotetext{
${ }^{6}$ In Van der Waerden notation these are written $\psi_{\alpha}$ and $\bar{\chi}^{\dot{\alpha}}$. For our purposes it is sufficient to leave these indices implicit. It is sufficient to know that contractions $\chi \psi=\psi \chi$ and $\bar{\chi} \bar{\psi}=\bar{\psi} \bar{\chi}$ are allowed, but $\chi \bar{\psi}$ and $\bar{\chi} \psi$ are not.
} 
connect fermions of the same chirality:

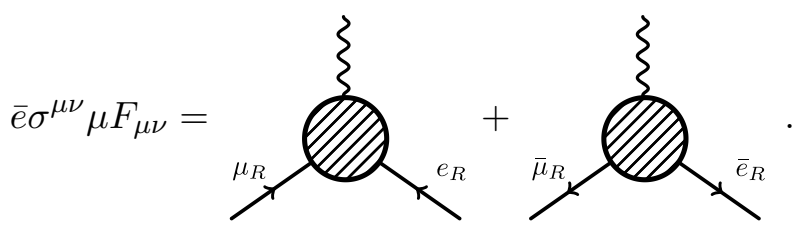

Because the lepton-flavor violating couplings of the $\varphi$ in (B.3)-(B.4), i.e. figure 7, dipole operators in the limit of a single off-diagonal flavor coupling can only connect states of opposite chirality and different flavor. There is no one-loop diagram with an internal $\varphi$ for this process because of the spurious $L_{e}-L_{\mu}$ symmetry. This symmetry is violated by the $W$ interactions so that the leading diagram must contain additionally a loop with a neutrino$W$ loop, as in the Standard Model process. One finds that the leading contribution to $\mu \rightarrow e \gamma$ from the $\varphi$ is suppressed by an additional loop factor compared to the already tiny Standard Model term.

Open Access. This article is distributed under the terms of the Creative Commons Attribution License (CC-BY 4.0), which permits any use, distribution and reproduction in any medium, provided the original author(s) and source are credited.

\section{References}

[1] ATLAS collaboration, Combined search for the standard model Higgs boson using up to $4.9 \mathrm{fb}^{-1}$ of pp collision data at $\sqrt{s}=7 \mathrm{TeV}$ with the ATLAS detector at the LHC, Phys. Lett. B 710 (2012) 49 [arXiv:1202.1408] [INSPIRE].

[2] CMS collaboration, Combined results of searches for the standard model Higgs boson in pp collisions at $\sqrt{s}=7 \mathrm{TeV}$, Phys. Lett. B 710 (2012) 26 [arXiv:1202.1488] [INSPIRE].

[3] D. Curtin et al., Exotic decays of the 125 GeV Higgs boson, Phys. Rev. D 90 (2014) 075004 [arXiv: 1312.4992] [INSPIRE].

[4] E. Izaguirre and D. Stolarski, Searching for Higgs decays to as many as 8 leptons, Phys. Rev. Lett. 121 (2018) 221803 [arXiv:1805.12136] [INSPIRE].

[5] M. Battaglieri et al., US cosmic visions: new ideas in dark matter 201\%: community report, FERMILAB-CONF-17-282 (2017).

[6] M. Pospelov, A. Ritz and M.B. Voloshin, Secluded WIMP dark matter, Phys. Lett. B 662 (2008) 53 [arXiv:0711.4866] [inSPIRE].

[7] J.L. Feng and J. Kumar, The WIMPless miracle: dark-matter particles without weak-scale masses or weak interactions, Phys. Rev. Lett. 101 (2008) 231301 [arXiv:0803.4196] [INSPIRE].

[8] J. Alexander et al., Dark sectors 2016 workshop: community report, arXiv:1608.08632 [INSPIRE].

[9] J.A. Evans, S. Gori and J. Shelton, Looking for the WIMP next door, JHEP 02 (2018) 100 [arXiv: 1712.03974] [INSPIRE].

[10] J.A. Evans, C. Gaidau and J. Shelton, Leak-in dark matter, arXiv:1909.04671 [INSPIRE].

[11] LHCb collaboration, Test of lepton universality using $B^{+} \rightarrow K^{+} \ell^{+} \ell^{-}$decays, Phys. Rev. Lett. 113 (2014) 151601 [arXiv: 1406.6482] [INSPIRE]. 
[12] LHCb collaboration, Measurement of the ratio of branching fractions $\mathcal{B}\left(\bar{B}^{0} \rightarrow D^{*+} \tau^{-} \bar{\nu}_{\tau}\right) / \mathcal{B}\left(\bar{B}^{0} \rightarrow D^{*+} \mu^{-} \bar{\nu}_{\mu}\right)$, Phys. Rev. Lett. 115 (2015) 111803 [Erratum ibid. 115 (2015) 159901] [arXiv:1506.08614] [INSPIRE].

[13] R. Pohl et al., The size of the proton, Nature 466 (2010) 213 [INSPIRE].

[14] Muon G-2 collaboration, Final report of the muon E821 anomalous magnetic moment measurement at BNL, Phys. Rev. D 73 (2006) 072003 [hep-ex/0602035] [InSPIRE].

[15] M. Pospelov, Secluded U(1) below the weak scale, Phys. Rev. D 80 (2009) 095002 [arXiv:0811.1030] [INSPIRE].

[16] V. Barger, C.-W. Chiang, W.-Y. Keung and D. Marfatia, Proton size anomaly, Phys. Rev. Lett. 106 (2011) 153001 [arXiv: 1011.3519] [INSPIRE].

[17] A. Datta, J. Liao and D. Marfatia, A light $Z^{\prime}$ for the $R_{K}$ puzzle and nonstandard neutrino interactions, Phys. Lett. B 768 (2017) 265 [arXiv:1702.01099] [INSPIRE].

[18] P. Asadi, M.R. Buckley and D. Shih, It's all right(-handed neutrinos): a new $W^{\prime}$ model for the $R_{D^{(*)}}$ anomaly, JHEP 09 (2018) 010 [arXiv: 1804.04135] [INSPIRE].

[19] A. Greljo, D.J. Robinson, B. Shakya and J. Zupan, $R\left(D^{(*)}\right)$ from $W^{\prime}$ and right-handed neutrinos, JHEP 09 (2018) 169 [arXiv: 1804.04642] [INSPIRE].

[20] D.J. Robinson, B. Shakya and J. Zupan, Right-handed neutrinos and $R\left(D^{(a s t)}\right)$, JHEP 02 (2019) 119 [arXiv: 1807.04753] [INSPIRE].

[21] SINDRUM collaboration, Search for the decay $\mu^{+} \rightarrow e^{+} e^{+} e^{-}$, Nucl. Phys. B 299 (1988) 1 [INSPIRE].

[22] BABAR collaboration, Searches for lepton flavor violation in the decays $\tau^{ \pm} \rightarrow e^{ \pm} \gamma$ and $\tau^{ \pm} \rightarrow \mu^{ \pm} \gamma$, Phys. Rev. Lett. 104 (2010) 021802 [arXiv:0908.2381] [INSPIRE].

[23] I. Galon, A. Kwa and P. Tanedo, Lepton-flavor violating mediators, JHEP 03 (2017) 064 [arXiv: 1610.08060] [INSPIRE].

[24] L. Goodenough and D. Hooper, Possible evidence for dark matter annihilation in the inner Milky Way from the Fermi Gamma Ray Space Telescope, arXiv:0910.2998 [INSPIRE].

[25] D. Hooper and L. Goodenough, Dark matter annihilation in the Galactic Center as seen by the Fermi Gamma Ray Space Telescope, Phys. Lett. B 697 (2011) 412 [arXiv:1010.2752] [INSPIRE].

[26] K.N. Abazajian, The consistency of Fermi-LAT observations of the Galactic Center with a millisecond pulsar population in the Central Stellar Cluster, JCAP 03 (2011) 010 [arXiv: 1011.4275] [INSPIRE].

[27] A. Boyarsky, D. Malyshev and O. Ruchayskiy, A comment on the emission from the Galactic Center as seen by the Fermi telescope, Phys. Lett. B 705 (2011) 165 [arXiv: 1012.5839] [INSPIRE].

[28] D. Hooper and T. Linden, On the origin of the gamma rays from the Galactic Center, Phys. Rev. D 84 (2011) 123005 [arXiv:1110.0006] [INSPIRE].

[29] K.N. Abazajian and M. Kaplinghat, Detection of a gamma-ray source in the Galactic Center consistent with extended emission from dark matter annihilation and concentrated astrophysical emission, Phys. Rev. D 86 (2012) 083511 [Erratum ibid. D 87 (2013) 129902] [arXiv:1207.6047] [INSPIRE].

[30] C. Gordon and O. Macias, Dark matter and pulsar model constraints from Galactic Center Fermi-LAT gamma ray observations, Phys. Rev. D 88 (2013) 083521 [Erratum ibid. D 89 (2014) 049901] [arXiv:1306.5725] [INSPIRE]. 
[31] D. Hooper and T.R. Slatyer, Two emission mechanisms in the Fermi bubbles: a possible signal of annihilating dark matter, Phys. Dark Univ. 2 (2013) 118 [arXiv:1302.6589] [INSPIRE].

[32] W.-C. Huang, A. Urbano and W. Xue, Fermi bubbles under dark matter scrutiny. Part I: astrophysical analysis, arXiv:1307.6862 [INSPIRE].

[33] N. Okada and O. Seto, Gamma ray emission in Fermi bubbles and Higgs portal dark matter, Phys. Rev. D 89 (2014) 043525 [arXiv:1310.5991] [INSPIRE].

[34] O. Macias and C. Gordon, Contribution of cosmic rays interacting with molecular clouds to the Galactic Center gamma-ray excess, Phys. Rev. D 89 (2014) 063515 [arXiv:1312.6671] [INSPIRE].

[35] K.N. Abazajian, N. Canac, S. Horiuchi and M. Kaplinghat, Astrophysical and Dark Matter interpretations of extended gamma-ray emission from the Galactic Center, Phys. Rev. D 90 (2014) 023526 [arXiv: 1402.4090] [INSPIRE].

[36] T. Daylan et al., The characterization of the gamma-ray signal from the central Milky Way: a case for annihilating dark matter, Phys. Dark Univ. 12 (2016) 1 [arXiv:1402.6703] [INSPIRE].

[37] B. Zhou et al., GeV excess in the Milky Way: the role of diffuse galactic gamma-ray emission templates, Phys. Rev. D 91 (2015) 123010 [arXiv:1406.6948] [INSPIRE].

[38] F. Calore, I. Cholis and C. Weniger, Background model systematics for the Fermi GeV excess, JCAP 03 (2015) 038 [arXiv:1409.0042] [INSPIRE].

[39] F. Calore, I. Cholis, C. McCabe and C. Weniger, A tale of tails: dark matter interpretations of the Fermi GeV excess in light of background model systematics, Phys. Rev. D 91 (2015) 063003 [arXiv: 1411.4647] [INSPIRE].

[40] F. Calore, I. Cholis and C. Weniger, The GeV excess shining through: background systematics for the inner galaxy analysis, arXiv:1502.02805 [INSPIRE].

[41] Fermi-LAT collaboration, Fermi-LAT observations of high-energy $\gamma$-ray emission toward the Galactic Center, Astrophys. J. 819 (2016) 44 [arXiv:1511.02938] [INSPIRE].

[42] R.K. Leane and T.R. Slatyer, Dark matter strikes back at the Galactic Center, arXiv: 1904.08430 [INSPIRE].

[43] L.J. Chang et al., Characterizing the nature of the unresolved point sources in the Galactic Center, arXiv: 1908.10874 [INSPIRE].

[44] N. Craig et al., The unexplored landscape of two-body resonances, Acta Phys. Polon. B 50 (2019) 837 [arXiv: 1610.09392] [inSPIRE].

[45] J.H. Kim, K. Kong, B. Nachman and D. Whiteson, The motivation and status of two-body resonance decays after the LHC Run 2 and beyond, arXiv:1907.06659 [INSPIRE].

[46] I. Galon and J. Zupan, Dark sectors and enhanced $h \rightarrow \tau \mu$ transitions, JHEP 05 (2017) 083 [arXiv: 1701.08767] [INSPIRE].

[47] J. Heeck and W. Rodejohann, Lepton flavor violation with displaced vertices, Phys. Lett. B 776 (2018) 385 [arXiv:1710.02062] [InSPIRE].

[48] P.S.B. Dev, R.N. Mohapatra and Y. Zhang, Lepton flavor violation induced by a neutral scalar at future lepton colliders, Phys. Rev. Lett. 120 (2018) 221804 [arXiv:1711.08430] [INSPIRE].

[49] J. Kile and A. Soni, Flavored dark matter in direct detection experiments and at LHC, Phys. Rev. D 84 (2011) 035016 [arXiv:1104.5239] [INSPIRE]. 
[50] B. Batell, J. Pradler and M. Spannowsky, Dark matter from minimal flavor violation, JHEP 08 (2011) 038 [arXiv:1105.1781] [INSPIRE].

[51] J.F. Kamenik and J. Zupan, Discovering dark matter through flavor violation at the LHC, Phys. Rev. D 84 (2011) 111502 [arXiv:1107.0623] [INSPIRE].

[52] P. Agrawal, S. Blanchet, Z. Chacko and C. Kilic, Flavored dark matter and its implications for direct detection and colliders, Phys. Rev. D 86 (2012) 055002 [arXiv:1109.3516] [INSPIRE].

[53] CMS collaboration, Searches for R-parity-violating supersymmetry in ppcollisions at $\sqrt{s}=8$ TeV in final states with 0-4 leptons, Phys. Rev. D 94 (2016) 112009 [arXiv: 1606.08076] [INSPIRE].

[54] B. Batell, A. Freitas, A. Ismail and D. Mckeen, Flavor-specific scalar mediators, Phys. Rev. D 98 (2018) 055026 [arXiv: 1712.10022] [InSPIRE].

[55] O. Gedalia and G. Perez, Flavor physics, in the proceedings of DOIPhysics of the large and the small (TASI 09), June 1-26, Boulder, U.S.A. (2009), arXiv:1005.3106 [INSPIRE].

[56] Y. Grossman and P. Tanedo, Just a taste: lectures on flavor physics, in the proceedings of Anticipating the Next Discoveries in Particle Physics (TASI 2016), June 6-July, Boulder, U.S.A. (2016), arXiv:1711.03624 [INSPIRE].

[57] R.H. Bernstein and P.S. Cooper, Charged lepton flavor violation: an experimenter's guide, Phys. Rept. 532 (2013) 27 [arXiv: 1307.5787] [INSPIRE].

[58] L. Willmann et al., New bounds from searching for muonium to anti-muonium conversion, Phys. Rev. Lett. 82 (1999) 49 [hep-ex/9807011] [INSPIRE].

[59] J.E. Kim, P. Ko and D.-G. Lee, More on R-parity and lepton family number violating couplings from muon(ium) conversion and $\tau$ and $\pi^{0}$ decays, Phys. Rev. D 56 (1997) 100 [hep-ph/9701381] [INSPIRE].

[60] P. Langacker, Precision tests of standard model of electroweak interactions, World Scientific, Singapore (1996).

[61] V.D. Barger, G.F. Giudice and T. Han, Some new aspects of supersymmetry R-parity violating interactions, Phys. Rev. D 40 (1989) 2987 [INSPIRE].

[62] R. Barbier et al., R-parity violating supersymmetry, Phys. Rept. 420 (2005) 1 [hep-ph/0406039] [INSPIRE].

[63] R. Harnik, J. Kopp and J. Zupan, Flavor violating Higgs decays, JHEP 03 (2013) 026 [arXiv: 1209.1397] [INSPIRE].

[64] MEG collaboration, Search for the lepton flavour violating decay $\mu^{+} \rightarrow e^{+} \gamma$ with the full dataset of the MEG experiment, Eur. Phys. J. C 76 (2016) 434 [arXiv:1605.05081] [INSPIRE].

[65] BeLle-II collaboration, The Belle II physics book, arXiv:1808.10567 [INSPIRE].

[66] MEG II collaboration, The design of the MEG II experiment, Eur. Phys. J. C 78 (2018) 380 [arXiv: 1801.04688] [INSPIRE].

[67] K. Hayasaka et al., Search for lepton flavor violating $\tau$ decays into three leptons with 719 million produced $\tau^{+} \tau^{-}$pairs, Phys. Lett. B 687 (2010) 139 [arXiv:1001.3221] [INSPIRE].

[68] Mu3E collaboration, Status of the Mu3e experiment at PSI, EPJ Web Conf. 118 (2016) 01028 [arXiv: 1605. 02906] [INSPIRE]. 
[69] CMS collaboration, Search for new phenomena with multiple charged leptons in proton-proton collisions at $\sqrt{s}=13$ TeV, Eur. Phys. J. C 77 (2017) 635 [arXiv: 1701.06940] [INSPIRE].

[70] CMS collaboration, Search for electroweak production of charginos and neutralinos in multilepton final states in proton-proton collisions at $\sqrt{s}=13$ TeV, JHEP 03 (2018) 166 [arXiv: 1709.05406] [INSPIRE].

[71] CMS collaboration, Combined search for electroweak production of charginos and neutralinos in proton-proton collisions at $\sqrt{s}=13$ TeV, JHEP 03 (2018) 160 [arXiv: 1801.03957] [INSPIRE].

[72] ATLAS collaboration, Search for supersymmetry in events with four or more leptons in $\sqrt{s}=13$ TeV pp collisions with ATLAS, Phys. Rev. D 98 (2018) 032009 [arXiv: 1804.03602] [INSPIRE].

[73] CMS collaboration, Search for vector-like leptons in multilepton final states in proton-proton collisions at $\sqrt{s}=13$ TeV, Phys. Rev. D 100 (2019) 052003 [arXiv: 1905.10853] [INSPIRE].

[74] D0 collaboration, Search for NMSSM Higgs bosons in the $h \rightarrow$ aa $\rightarrow \mu \mu \mu \mu, \mu \mu \tau \tau$ channels using p $\bar{p}$ collisions at $\sqrt{s}=1.96$ TeV, Phys. Rev. Lett. 103 (2009) 061801 [arXiv:0905.3381] [INSPIRE].

[75] ATLAS collaboration, Search for Higgs bosons decaying to aa in the $\mu \mu \tau \tau$ final state in $p p$ collisions at $\sqrt{s}=8$ TeV with the ATLAS experiment, Phys. Rev. D 92 (2015) 052002 [arXiv: 1505.01609] [INSPIRE].

[76] CMS collaboration, Search for light bosons in decays of the $125 \mathrm{GeV}$ Higgs boson in proton-proton collisions at $\sqrt{s}=8 \mathrm{TeV}$, JHEP 10 (2017) 076 [arXiv:1701.02032] [INSPIRE].

[77] ATLAS collaboration, Search for Higgs boson decays to beyond-the-Standard-Model light bosons in four-lepton events with the ATLAS detector at $\sqrt{s}=13 \mathrm{TeV}$, JHEP 06 (2018) 166 [arXiv: 1802.03388] [INSPIRE].

[78] A. Alloul et al., FeynRules 2.0 - A complete toolbox for tree-level phenomenology, Comput. Phys. Commun. 185 (2014) 2250 [arXiv:1310.1921] [INSPIRE].

[79] N.D. Christensen and C. Duhr, FeynRules — Feynman rules made easy, Comput. Phys. Commun. 180 (2009) 1614 [arXiv:0806.4194] [INSPIRE].

[80] J. Alwall et al., The automated computation of tree-level and next-to-leading order differential cross sections and their matching to parton shower simulations, JHEP $\mathbf{0 7}$ (2014) 079 [arXiv: 1405.0301] [INSPIRE].

[81] T. Sjöstrand et al., An introduction to PYTHIA 8.2, Comput. Phys. Commun. 191 (2015) 159 [arXiv: 1410.3012] [INSPIRE].

[82] M. Cacciari, G.P. Salam and G. Soyez, FastJet user manual, Eur. Phys. J. C 72 (2012) 1896 [arXiv:1111.6097] [INSPIRE].

[83] CMS collaboration, Search for RPV SUSY in the four-lepton final state, CMS-PAS-SUS-13-010 (2013).

[84] CMS collaboration, Performance of electron reconstruction and selection with the CMS detector in proton-proton collisions at $\sqrt{s}=8 \mathrm{TeV}, 2015$ JINST $10 \mathrm{P} 06005$ [arXiv: 1502.02701] [INSPIRE]. 
[85] CMS collaboration, Performance of the CMS muon detector and muon reconstruction with proton-proton collisions at $\sqrt{s}=13 \mathrm{TeV}, 2018$ JINST 13 P06015 [arXiv:1804.04528] [INSPIRE].

[86] M. Cacciari, G.P. Salam and G. Soyez, The anti-k $k_{t}$ jet clustering algorithm, JHEP 04 (2008) 063 [arXiv: 0802.1189] [inSPIRE].

[87] Particle Data Group, Status of Higgs boson physics, RPP2018-REV-HIGGS-BOSON (2019).

[88] CMS collaboration, Measurement of inclusive $W$ and $Z$ boson production cross sections in pp collisions at $\sqrt{s}=8 \mathrm{TeV}$, Phys. Rev. Lett. 112 (2014) 191802 [arXiv:1402.0923] [INSPIRE].

[89] R.D. Cousins and V.L. Highland, Incorporating systematic uncertainties into an upper limit, Nucl. Instrum. Meth. A 320 (1992) 331.

[90] T. Junk, Confidence level computation for combining searches with small statistics, Nucl. Instrum. Meth. A 434 (1999) 435.

[91] A. L. Read, Presentation of search results: the CL $L_{s}$ technique, J. Phys. G 28 (2002) 2693.

[92] Particle Data Group collaboration, Review of particle physics, Phys. Rev. D 98 (2018) 030001 [INSPIRE].

[93] CMS collaboration, Performance of reconstruction and identification of $\tau$ leptons decaying to hadrons and $\nu_{\tau}$ in pp collisions at $\sqrt{s}=13 \mathrm{TeV}, 2018$ JINST $13 \mathrm{P} 10005$

[arXiv: 1809.02816] [INSPIRE].

[94] M. Grazzini, S. Kallweit, M. Wiesemann and J.Y. Yook, ZZ production at the LHC: NLO QCD corrections to the loop-induced gluon fusion channel, JHEP 03 (2019) 070 [arXiv: 1811.09593] [INSPIRE].

[95] J.A. Evans and J. Shelton, Long-lived staus and displaced leptons at the LHC, JHEP 04 (2016) 056 [arXiv: 1601.01326] [INSPIRE].

[96] J. Alimena et al., Searching for long-lived particles beyond the standard model at the Large Hadron Collider, arXiv: 1903.04497 [INSPIRE].

[97] CMS collaboration, Search for displaced supersymmetry in events with an electron and a muon with large impact parameters, Phys. Rev. Lett. 114 (2015) 061801 [arXiv:1409.4789] [INSPIRE].

[98] ATLAS collaboration, Search for massive, long-lived particles using multitrack displaced vertices or displaced lepton pairs in pp collisions at $\sqrt{s}=8 \mathrm{TeV}$ with the ATLAS detector, Phys. Rev. D 92 (2015) 072004 [arXiv:1504.05162] [INSPIRE].

[99] ATLAS collaboration, Search for displaced vertices of oppositely charged leptons from decays of long-lived particles in pp collisions at $\sqrt{s}=13 \mathrm{TeV}$ with the ATLAS detector, arXiv: 1907.10037 [INSPIRE].

[100] CMS collaboration, Search for long-lived particles that decay into final states containing two electrons or two muons in proton-proton collisions at $\sqrt{s}=8 \mathrm{TeV}$, Phys. Rev. D 91 (2015) 052012 [arXiv:1411.6977] [INSPIRE].

[101] ATLAS collaboration, Search for long-lived neutral particles decaying into lepton jets in proton-proton collisions at $\sqrt{s}=8 \mathrm{TeV}$ with the ATLAS detector, JHEP 11 (2014) 088 [arXiv: 1409.0746] [INSPIRE]. 
[102] ATLAS collaboration, Search for heavy neutral leptons in decays of $W$ bosons produced in $13 \mathrm{TeV}$ pp collisions using prompt and displaced signatures with the ATLAS detector, JHEP 10 (2019) 265 [arXiv: 1905.09787] [inSPIRE].

[103] C.D. Froggatt and H.B. Nielsen, Hierarchy of quark masses, Cabibbo angles and CP-violation, Nucl. Phys. B 147 (1979) 277 [INSPIRE].

[104] A. Halprin and A. Masiero, Muonium-anti-muonium oscillations and exotic muon decay in broken R-parity SUSY models, Phys. Rev. D 48 (1993) R2987.

[105] R.N. Mohapatra, Rare muon decays and physics beyond the Standard Model, Z. Phys. C56 (1992) S117.

[106] H. Dreiner, An introduction to explicit R-parity violation, Adv. Ser. Direct. High Energy Phys. 21 (2010) 565 [hep-ph/9707435].

[107] Y. Grossman and H.E. Haber, (S)neutrino properties in R-parity violating supersymmetry. 1. CP conserving phenomena, Phys. Rev. D 59 (1999) 093008 [hep-ph/9810536] [InSPIRE].

[108] S.P. Martin, TASI 2011 lectures notes: two-component fermion notation and supersymmetry, in the proceedings of The Dark Secrets of the Terascale (TASI 2011), June 6-July 11, Boulder, U.S.A. (2011), arXiv: 1205.4076 [INSPIRE].

[109] H.K. Dreiner, H.E. Haber and S.P. Martin, Two-component spinor techniques and Feynman rules for quantum field theory and supersymmetry, Phys. Rept. 494 (2010) 1 [arXiv:0812.1594] [INSPIRE]. 